Canadian University Music Review

Canadian University Music Review

Revue de musique des universités canadiennes

\title{
La Cathédrale engloutie de Claude Debussy : de la perception au sens
}

\section{Michel Imberty}

Numéro 6, 1985

URI : https://id.erudit.org/iderudit/1014034ar

DOI : https://doi.org/10.7202/1014034ar

Aller au sommaire du numéro

Éditeur(s)

Canadian University Music Society / Société de musique des universités canadiennes

ISSN

0710-0353 (imprimé)

2291-2436 (numérique)

Découvrir la revue

Citer cet article

Imberty, M. (1985). La Cathédrale engloutie de Claude Debussy : de la perception au sens. Canadian University Music Review / Revue de musique des universités canadiennes, (6), 90-160. https://doi.org/10.7202/1014034ar

(C) Canadian University Music Society / Société de musique des universités canadiennes, 1985
Ce document est protégé par la loi sur le droit d'auteur. L'utilisation des services d'Érudit (y compris la reproduction) est assujettie à sa politique d'utilisation que vous pouvez consulter en ligne.

https://apropos.erudit.org/fr/usagers/politique-dutilisation/ 


\title{
LA CATHÉDRALE ENGLOUTIE DE CLAUDE DEBUSSY: DE LA PERCEPTION AU SENS
}

\author{
Michel Imberty
}

D'une façon générale, on peut dire que la démarche des commentateurs de la Cathédrale engloutie est toujours la même : le titre indiquant qu'il s'agit de la légende de la ville d'Ys mise en musique, les descriptions sémantiques consistent à retrouver les épisodes de la légende dans les principaux moments de la pièce musicale. À l'inverse, l'étude expérimentale qui suit, tente d'aborder l'analyse sémantique au niveau esthésique, c'est-à-dire à partir des significations spontanément reconnues par des auditeurs " naïfs », ne connaissant pas le titre de l'oeuvre.

Cependant, le secteur s'apercevra vite que l'objet essentiel de l'étude n'est pas là. En effet, il n'est peut-être pas de grand intérêt de savoir si les auditeurs ne connaissant pas l'oeuvre y retrouvent l'atmosphère de la vielle légende bretonne, tant qu'on demeure incapable de dire en quoi consiste, pour eux, la structure de la pièce musicale, de dire ce qu'ils en ont retenu, et pourquoi elle les a touchés au point que certaines associations imaginatives se sont développées selon les grandes lignes de la légende - ou au contraire peut-être, en dehors d'elle.

Autrement dit, l'analyse sémantique ne peut guère avoir de sens sans une analyse esthésique structurale qui fournisse quelques indications sur le décodage perceptif de la pièce musicale lors de l'écoute. C'est cette analyse-là qui constitue l'essentiel de cette étude.

\section{Le style musical dans les études sémiologiques}

En fait, comme on le verra plus loin, nous allons essayer d'aborder, à travers l'expérimentation psychologique, le problème du style de Debussy dans la Cathédrale engloutie. L'entreprise ne se justifiera que progressivement, et nous demandons seulement 
au lecteur d'admettre au moins provisoirement que le style peut étre défini en terme de structure, c'est-à-dire en terme de rapports d'unités ou d'éléments au sein d'une totalité organique, l'oeuvre analysée et/ou écoutée. Mais comment rendre compte de cette structure?

Dans un ouvrage récent, Deliège (1984) envisage de manière critique plusieurs approches possibles du style musical. Selon l'auteur, il est d'abord possible d'envisager le style sous forme d'ensembles plus ou moins précis de traits généraux communs à des groupes d'oeuvres ou aux oeuvres d'un compositeur déterminé que, par comparaison, on ne retrouvera pas dans d'autres groupes d'oeuvres ou chez d'autres compositeurs. Ici, la notion de style englobe aussi bien ce qu'on peut appeler style d'auteur, style d'école ou style d'époque. Cette approche du style risque de rester très incomplète et extérieure, car elle ne retient précisément que ce qu'il y a de plus banal et de plus évident, alors que le style se décrit parfois aussi bien dans un geste idiosyncrasique rare qui signe l'oeuvre.

C'est sans doute au même genre de critiques que se trouverait soumise une conception purement taxinomique ou statistique du style: celui-ci pourrait en effet être décrit, soit par des recensements de traits à l'intérieur d'un système codifié donné, ces traits témoignant des choix d'un compositeur par rapport aux possibilités proposées par le système ; soit par des recensements d'écarts par rapport aux usages les plus fréquents ou aux normes codifiées par une époque ou une culture. Dans les deux cas, on devrait aboutir, après un long travail, à une sorte de tableau des effets de style relevés dans les oeuvres. Mais dans une telle perspective, l'explication reste hors d'atteinte, les taxinomies stylistiques étant, par définition sans doute, inachevées.

Deliège oppose à cette approche ce qu'il appele les " conceptions idéalistes " (Deliège $1984: 241$ ) : celles-ci lient le style au goût. Avoir du style, c'est d'abord savoir surprendre par un raffinement formel et expressif qui ne saurait se réduire à des lois. Deliège rejette naturellement cette intrusion de l'esthétique dans la st ylistique, car, selon lui, le style, pour donner lieu à une théorie et à une description exhaustive, ne doit pas être amagalmé à l'investigation des valeurs : "Nous avons beau savoir que le style de Debussy nous sollicite tout autrement que la musique du bal musette, la stylistique l'ignore." (ibid. : 242). La stylistique, comme la syntaxe, se doit d'être une théorie générale et un modèle ; elle doit avoir une méthodologie d'analyse rigoureuse et répétable, 
bref, elle ne peut et ne doit départager qu'au plan de la modélisation des structures, non au plan du jegement.

C'est pourquoi Deliège en vient à une conception apparemment structurale. Apparemment, car ce qu'il considère comme objet initial de toute stylistique musicale, c'est le phénomène de la forme qu'il réfère explicitement à la Gestalttheorie et à ses présupposés psychologiques. Or la Gestaltheorie n'est pas une théorie de la structure, au sens où la linguistique et la sémiologie l'entendent aujourd'hui.

La forme est une totalité organisée où les éléments ne valent que par les réseaux de relations qu'ils entretiennent. La forme n'est pas une concaténation de ces éléments, leur organisation seule la définit. Les nombreuses références de Deliège à l'ouvrage déjà ancien de Köhler (Gestalt Psychology, 1929), montrent la séduction qu'exerce sur l'auteur l'idée de totalité qu'il transpose à l'oeuvre musicale. Précisons : le travail de Deliège est centré sur la syntaxe, et la théorie schenkérienne dont il s'inspire implique que les structures musicales sont engendrées à partir de " formants ... concaténés, bien connectés dans leur mode de superposition " (Deliège 1984 : 247). Mais Deliège pense que pour aborder le style, il faut passer au niveau de la forme. Or pour lui, il est clair que la forme " concrète " suppose à la fois des structures engendrées par les règles d'un code, et des éléments surajoutés, " non codés ", qui précisément font le style, non à eux seuls, mais par les modifications qualitatives qu'ils introduisent dans le code.

Reprenant le point de vue de Granger (1979: 133 et sq.), Deliège parvient donc à une conception du style à la fois totalisante et dialectique, impliquant dans l'analyse, l'articulation d'éléments pertinents codés et d'éléments non pertinents, extérieurs au code. "Le style de l'oeuvre s'exprimerait dans le mode d'utilisation par l'auteur des éléments relevant du système de référence joints à la manière qui lui est propre de recourir à ceux qui n'en relèvent pas " (Deliège $1984: 248$ ).

C'est cela la forme concrète (expression que Deliège reprend à Bachelard et à Ruyer), cette structure d'ensemble de l'oeuvre non réductible à la syntaxe, où se dévoilent à la fois les traits généraux et le geste rare, signature parfois unique de l'oeuvre, lu « autant dans la norme que dans l'écart, autant dans l'attendu que le surprenant » (ibid.: 251).

Deux difficultés cependant surgissent : une difficulté méthodologique que Deliège n'esquive pas ; une difficulté théorique plus profonde qui provient de la dualité de points de vue adoptée. La 
difficulté méthodologique d'abord: comment saisir et décrire exhaustivement, objectivement, cette totalité complexe, cette forme à la fois première et finale où se dessine le style ? Deliège reconnaît qu'il y a un écueil, car si la description structurale de la couche morphologique et syntaxique peut se concevoir selon des procédures sémiologiques bien éprouvées, il n'en va pas de même pour ce qui n'est pas pertinent par rapport au code de l'infrastructure, de ce qui est par essence imprévisible, inattendu. Le risque est grand de se laisser entraîner à la seule intuition du musicologue, dans une analyse sans critères explicites, repérant tout au hasard des impressions subjectives, des modes, des nécessités d'une polémique ; ou à l'inverse, le risque est tout aussi réel de passer à côté du détail révélateur, de n'avoir qu'une vue très superficielle et extérieure de la totalité dynamique de l'oeuvre. L'idéal est bien sûr de pratiquer une méthode d'analyse qui repose sur un " mouvement dialectique " oscillant " entre le général et le particulier " (ibid.: 249). Mais Deliège reconnaît que le succès d'une telle analyse n'est pas totalement assuré. Or curieusement, l'auteur ne semble guère vouloir se donner les moyens pour éviter les écueils du flou méthodologique qu'il aperçoit : il rejette toute tentative d'inventaire exhaustif fondé sur une observation statistique des faits de style. Selon lui, la statistique pêcherait par excès de subjectivisme! Le degré de vraisemblance qu'elle introduit ne serait qu'illusion, car elle conduirait systématiquement à écarter ce qui est rare, et par là signifiant. La description statistique ne saurait atteindre cette merveilleuse - et un peu mystérieuse - totalité qui, seule, dévoile le style.

Il convient sur ce point d'être plus nuancé : la structure totale, cette forme concrète que cherche à atteindre Deliège, n'est certes pas une simple addition d'éléments dénombrables. Les relations de toute sorte et à tous les niveaux priment. Cependant, il ne faudrait pas confondre les faits eux-mêmes - sur la nature desquels on peut être provisoirement d'accord avec Deliège - et les méthodes d'investigation pour collationner ces faits et vérifier certaines hypothèses formulées à leur égard. Au plan de la syntaxe déjà, la remarque porte : je n'ai certes rien dit de la fonction de tonique dans une séquence en do majeur lorsque j'ai constaté la fréquence élevée de la note do, mais cette fréquence est un indice contrôlable sérieux pour cerner l'existence d'une tonique et certains de ses aspects fonctionnels. On sait d'ailleurs que les séquences tonales (dans un sens large) sont à forte redondance interne (en termes de théorie de l'information), et, suivant les systèmes musicaux 
utilisés, les intervalles pivôts sont eux-mêmes fortement redondants (faible quantité d'information). La même remarque vaut pour le style : je n'ai certes pas dit grand chose du style de Debussy lorsque j'ai constaté la fréquence des enchaînements parallèles d'accords de neuvième naturelle, mais la fréquence de ces enchaînements est un bon indice de certaines structures harmoniques de Debussy. A preuve d'ailleurs qu'un pastiche debussyste comporterait à coup sûr au moins une série de neuvièmes parallèles ! Bref, comme le notait déjà Nattiez (1975), toute théorie ou toute construction d'un modèle abstrait suppose d'abord une prise d'information aussi complète que possible, donc l'établissement de taxinomies, et la démarche empirique - qui s'appuie nécessairement sur des données quantitatives - n'est pas incompatible avec la démarche inverse qui part d'hypothèses théoriques, schenkériennes ou autres, construit un modèle par élaboration hypothético-déductive, et retourne au "corpus " seulement pour vérifier ou falsifier le modèle.

La seconde difficulté est, nous l'avons dit, d'ordre théorique. En effet, une théorie du style, selon Deliège, doit en somme s'articuler en deux niveaux : celui du code, celui du non-code, c'est-à-dire du domaine de l'expression et du goût. Le style, c'est le résultat de cet échafaudage complexe dans une forme. Or Deliège se réfère ainsi explicitement à deux conceptions radicalement opposées de la structure : d'une part, au niveau du code, une conception générative délibérément chomskyenne avec toutes ses implications au plan psychologique ; d'autre part, au niveau du non-code, une conception globalisante et perceptive de la structure - qui désormais s'appelle forme -, entièrement statique, et qui relève de soi-disant lois "naturelles" d'auto-répartition des stimulations tant aux entrées des organes sensoriels que dans les zones corticales correspondantes. Outre que cette hypothèse s'est avérée fausse depuis longtemps au plan neurophysiologique, elle est en totale contradiction avec l'idée chomskyenne de compétence du sujet, sur laquelle repose la conception du niveau syntaxique développée par Deliège.

La contradiction interne à la thèse de Deliège provient en réalité d'une double insuffisance de son analyse : au niveau structural et au niveau psychologique. Au niveau structural, il nous semble que c'est lorsque Deliège aborde ce qu'il appelle la structure prosodique de l'oeuvre, qu'il dévie de la conception initiale. Il retient comme " unité, formant ou figure " (1984:177) le groupe élan, sommet, chute (anacrouse, accent, désinence) 
longuement décrit par Messiaen. Or Deliège rattache explicitement, y compris par le terme utilisé de figure, cette unité à un phénomène de perception tels que ceux décrits par la Gestalttheorie. Mais pourquoi avoir recours aux résultats anciens et triviaux d'une théorie de la perception bien caduque pour rendre compte d'une structure dont, a priori, on ne voit pas pourquoi elle ne relèverait pas d'un système de règles dérivé lui-même du système syntaxique ? Pourquoi faut-il avoir recours à l'illusion d'une théorie de l'auto-répartition des stimulations, dont on sait qu'elle ne correspond à aucun phénomène neurophysiologique réel ? Faut-il ajouter que bien des hypothèses de Köhler - traduit en France, il est vrai, seulement en 1964 - sur les soi-disant correspondances entre la perception visuelle de l'espace et la perception temporelle, relèvent de phénomènes fortement culturalisés et non de lois universelles ? D'ailleurs, les conclusions du chapitre consacré à cette question montrent combien, dès que l'on quitte le système tonal le plus orthodoxe, les difficultés pour retrouver cette figure primordiale s'accumulent ... C'est une contradiction de plus entre la prétendue universalité des lois de la Gestalt theorie et la complexité des faits que Deliège sait fort bien par ailleurs nous exposer.

C'est qu'en réalité, l'objet du livre de Deliège n'est pas l'étude du style: il le précise lui-même, s'en tenant à une théorie syntaxique de la musique tonale qu'il situe d'emblée dans la perspective générativiste. Il s'agit donc bien d'une théorie de la compétence musicale capable de rendre compte de toutes les structures et processus qui relèvent de ce code. Les limites de l'ouvrage étant fixées, la démarche est précise, vigoureuse, souvent convaincante. Mais, on l'a vu, Deliège se rend bien compte qu'une théorie du style ne peut être ramenée à une théorie syntaxique. D'où l'échappatoire recherchée, qui laisse perplexe.

Avant lui, dans un article riche mais polémique, Ruwet (1975) avait tenté de pousser la démarche jusqu'en ses ultimes conséquences. Ruwet partait d'une constatation - sur laquelle nous allons revenir, car elle est d'une extrême importance - à savoir que tout bon musicien reconnaît aisément (et cela dès les premières mesures) que telle sonate est de Mozart, telle autre de Beethoven ou de Schubert. Si le style n'était que le résultat de certaines répétitions d'événements sonores reconnus pertinents par leur fréquence, il faudrait à l'auditeur beaucoup plus de temps pour identifier le style du compositeur que ne le suppose l'expérience quotidienne. C'est donc que cette identification relève 
d'une capacité générale engendrant elle-même ses propres règles de production et de perception des processus musicaux.

La création musicale résulterait d'abord d'une certaine capacité à engendrer certaines structures sonores et non d'autres, et, en appliquant au modèle des contraintes suffisantes, on devrait pouvoir obtenir des structures d'un style potentiellement défini. Potentiellement : on veut dire par-là qu'il serait possible d'engendrer - au moins en partie, sinon dans le détail - de nouvelles productions musicales à l'intérieur des contraintes d'un style. Ruwet, toujours dans le même article (1975), imagine que Stravinsky soit mort en 1938 : une théorie du style de Stravinsky devrait permettre de situer tout Stravinsky, y compris le Stravinsky potentiel en 1938 de Agon, par rapport à d'autres productions musicales. "Il n'est pas déraisonnable d'espérer qu'une théorie, qui tiendrait compte à la fois des oeuvres connues de Stravinsky $(\ldots)$ et de règles générales de l'organisation musicale, et qui ne reculerait pas devant les hypothèses hardies, soit en mesure de déterminer quel type de musique Stravinsky, en aucun cas, n'aurait pu écrire, et du coup, de délimiter un certain champ à l'intérieur duquel Agon, etc... sont possibles " (Ruwet 1975 : 21-22). On voit le principe de la démarche : élaborer un modèle trés général qui, particularisé par certaines contraintes, engendrerait tout Stravinsky, et du même coup, nous serions en possession d'une " explication " du style de Stravinsky.

Un des sous-entendus de cette démarche est qu'il existe une sorte de compétence-noyau universelle, à l'intérieur de laquelle viennent se placer les règles de fonctionnement particulières à chaque style, chaque époque ou chaque civilisation. Mais en même temps, cette compétence-noyau expliquerait pourquoi l'auditeur identifie le style de Stravinsky dès les premières mesures de Agon ou du Sacre, tout comme il identifie le style de Mozart, le style baroque ou le style romantique. En même temps que capacité à générer des structures musicales en nombre infini, cette compétence serait - et peut-être plus encore - capacité à recevoir, à décoder ces structures et leurs différentes contraintes spécifiques, mais dont les lois très générales et universelles pourraient être mises en évidence.

Le modèle suggéré par Ruwet procède donc par spécifications successives, et le style n'est plus qu'une réalisation particulière, particulièrement contrainte de la compétence, alors que pour Deliège le style est à la fois en prolongement des règles syntaxiques et extérieur à ces règles. Mais le modèle de Ruwet est 
sans doute encore plus problématique que celui de Deliège : les essais de simulation par ordinateur ont montré combien alors le système d'instructions engendrant du Schumann ou du Bach ne pouvait être construit sans le recours préalable à des recensements nombreux, statistiques ou non, sur des corpus spécialement choisis.

\section{Pour une approche perceptive du style}

En réalité, Deliège s'est engagé dans la bonne voie, en cherchant à aborder le style à travers des concepts empruntés à la psychologie de la perception, mais il s'est trompé en développant une conception globalisante de la forme qui ne pouvait guère servir qu'à réaffirmer quelques évidences. En outre, il semble éprouver encore quelques réticences - comme tout musicologue - à penser que la réalité perceptive (ce que Nattiez appelle le niveau esthésique de l'oeuvre) ait quelque importance pour saisir la structure stylistique. Après tout, Francès (1958) a bien montré jadis quels décalages peuvent exister entre " structure perçue » et "structure conçue ".

Cependant, les expériences de cet auteur sont déjà très significatives : à propos de l'identification de thèmes dans les formes classiques, sonate et fugue, il montre que les erreurs dues aux fausses reconnaissances ou aux non reconnaissances sont importantes, même chez les sujets musiciens : des processus d'assimilation entrent en jeu qui aboutissent à une réorganisation de la forme conçue en fonction des capacités de différenciation perceptive des sujets. Et Francès conclut : " [la perception totale de l'oeuvre] est conditionnée sans doute par la quantité d'éléments sensoriels que l'auditeur peut embrasser à chaque instant, et par leur degré de présence ou de prégnance (...). Mais de cette richesse, l'auditeur qui cherche à comprendre l'unité d'ensemble, sa variété, le propre de l'invention, etc., abandonne une partie. L'intégration de la forme ne peut pas être une réintégration, mais une dialectique active et abstrayante " (Francès $1958: 247-48$ ).

Certes, on pourra répondre que l'identification des thèmes n'a peut-être que peu à voir avec l'organisation stylistique : "comprendre " la forme d'une oeuvre, en percevoir les éléments principaux, ce n'est pas pour autant reconnaître les caractéristiques d'un style. Alors que cette reconnaissance est, comme le dit Ruwet, très rapide, le processus d'identification des thèmes se déroule dans le temps, et il ne concerne que la forme " extérieure " et conventionnelle de l'oeuvre, c'est-à-dire le repérage d'une 
architecture vide dans laquelle vient se mouler plus ou moins bien l'oeuvre particulière. D'une certaine façon, toute oeuvre suppose des thèmes, des répétitions et des variations, des transpositions et des imitations de ces thèmes, qu'elles soient mélodiques, rythmiques, de timbre ou d'intensité, d'articulation, etc. De ce point de vue, les expériences de Francès sont d'abord des expériences portant sur la structuration d'un matériau perceptif, et non sur la perception d'une forme particulière illustrant un style.

Mais il serait absurde de penser que cette structuration est indépendante de l'accession au style, la forme "extérieure " de l'oeuvre d'une part étant considérablement remodelée à l'audition, le style de l'auteur d'autre part étant intrinsèquement et immédiatement accessible. L'organisation des thèmes, leurs successions, leurs répétitions, leurs développements, en même temps qu'ils constituent des faits très généraux - et généralement appris - qui fournissent des repères aux sujets, sont déjà, pour une oeuvre donnée, témoins d'une intention stylistique déterminée. Au fond, si tous les sujets ne perçoivent pas de la même façon les thèmes, ou plus généralement les grandes articulations d'une oeuvre, inversement, tous les compositeurs ne les organisent pas de la même façon. Et il n'y a aucune raison de penser que cette organisation conçue ne rejaillit pas sur l'organisation perçue. Par conséquent, le point de vue expérimental qui consiste à partir des conduites perceptives, n'exclut nullement la possibilité de revenir à la structure profonde de l'oeuvre, et il n'est pas inconcevable de pouvoir décrire certaines structures de style à partir des perceptions comparées d'oeuvres de compositeurs différents.

Francès montre d'ailleurs fort bien que la perception d'une oeuvre, à quelque niveau que ce soit, est un processus " dialectique " entre la réalité intrinsèque de la structure, et la réalité perceptive elle-même ; ainsi l'organisation abstraite de la structure, à laquelle font sans cesse référence Ruwet et Deliège, se double d'une organisation proprement perceptive dont les lois viennent se greffer sur les règles du système codé. Contrairement à ce que prétendait la Gestaltheorie, la perception n'est pas un décalque de la réalité, mais elle se constitue à travers des mécanismes complexes qui reconstruisent cette réalité à partir de données toujours fragmentaires fournies par les entrées sensorielles. Ces données sont en fait les plus saillantes du champ perceptif à l'instant $t$, et elles sont assimilées par les mécanismes qui les réélaborent. La réalité perçue dépend donc bien à la fois de ces mécanismes propres au sujet et de la configuration des stimuli à un 
moment donné du devenir sonore, les relations entre ces stimuli et ces mécanismes étant fonction notamment de la fréquence ou de la prégnance des premiers, de la disponibilité et du degré de généralité des seconds. Autrement dit, la structure - et spécialement la structure st ylistique - est à la fois le produit d'un ensemble de règles abstraites définissant des relations entre éléments sonores, et de lois de construction perceptive et cognitive qui concernent l'activité du sujet auditeur. Ainsi, il n'y aurait pas d'opposition radicale entre l'hypothèse d'une " compétence " ou " capacité de base "à structurer l'information qui rendrait compte de tous les phénomènes de décodage des organisations perceptives (reconnaissance de codes, de styles, de certaines qualités structurales simples comme le "lié-délié " d'une mélodie ...) et l'hypothèse d'apprentissage de certains modèles ou schémas très généraux qui seraient stockés en mémoire à partir des prises d'information à caractère probabiliste ou statistique sur le réel.

Une telle perspective devrait nous permettre de comprendre certains phénomènes bien connus des musiciens: dans son appréhension perceptive, le style dépend aussi de facteurs dynamiques qui peuvent, sous certaines conditions, le rendre méconnaissable pour l'auditeur. Ainsi, nous avons montré (Imberty 1979) que, dans une différenciation stylistique portant sur la comparaison de deux mélodies, l'une de Debussy, l'autre de Fauré, la modification du tempo entraîne une confusion des styles pour les auditeurs ne connaissant pas les oeuvres : le ralentissement de moitié du temps de Mandoline de Debussy la fait at tribuer à Fauré, alors que dans le tempo original la reconnaissaissance des styles se fait sans difficulté. C'est dire qu'en dehors des problèmes de formalisation des systèmes de règles, une théorie du style doit pouvoir répondre à des questions du genre de celles-ci : pourquoi (comme nous l'avons suggéré in Imberty 1974 et 1979) Debussy souffre-t-il, dans les mouvements lents, des interprétations en général beaucoup plus lentes que Brahms par exemple? $\mathrm{Ou}$ encore, pourquoi certaines interprétations paraissent-elles ternes, mièvres, sans intérêt, bref, " à côté " ? Quels rapports organiques le style entretient-il avec l'interprétation qu'on en donne, ou la perception que l'on en a ?

Dans un ordre d'idée assez proche d'ailleurs, Deliège, à son tour, pose des questions très pertinentes qui montrent l'intérêt d'envisager le style à travers sa perception. Par exemple, nous ne savons rien ou presque sur la façon dont nous effectuons les segmentations au cours de l'audition, et comment, ensuite, sont 
reliées en mémoire les séquences découpées dans la succession temporelle musicale. La question vaut autant pour les formes de durée brève que pour les oeuvres étalées sur des durées beaucoup plus longues ; et dans ce dernier cas, il est à parier qu'il y intervient des processus mentaux très spécifiques et sans doute dépendants de la structure elle-même.

Il paraît donc raisonnable de soutenir qu'il n'y a ni rupture complète, ni décalque exact entre l'intention créatrice et la réception de l'oeuvre par l'auditeur. Fondamentalement, l'hypothèse d'" universaux " cognitifs - et sans doute aussi affectifs permettant que l'oeuvre soit à la fois "communication " et "transmission" de message, est une hypothèse plausible, simplement parce que les processus qui aboutissent à la création de l'oeuvre sont en partie aussi ceux qui en permettent la compréhension. Mais en même temps, ce que montrent les expériences qui partent de l'esthésique, c'est que les distorsions sont aussi nombreuses que les concordances. Autrement dit, l'expérimentation à partir des conduites perceptive peut amener à certaines conclusions sur les structures sous-jacentes de l'oeuvre, mais ces conclusions doivent pouvoir être contrôlées dans une description exhaustive de la partition, elle-même fondée sur des procédures rigoureuses et explicites. L'intérêt du travail que nous présentons aujourd'hui sur la Cathédrale engloutie est de suggérer une confrontation des analyses du niveau neutre et du niveau esthésique de la même oeuvre. Ce qui n'interdit nullement, mais après, d'élaborer un modèle plus abstrait permettant des généralisations inductives, et pouvant peut-être nous renseigner sur les mécanismes de ce que Ruwet et Deliège appellent la « compétence musicale " .

\section{Problématique et hypothèses}

\section{A - Segmentation sémiologique et perception}

Tous les travaux de sémiologie musicale prennent comme point de départ de leur démarche une opération de segmentation du flux musical, et on peut dire, au moins dans un premier temps, que la structure d'une pièce musicale se dégage de cette segmentation réitérée à plusieurs niveaux. Ruwet (1972), avant d'abandonner cette perspective, l'avait parfaitement explicitée : considérer les unités les plus grandes possibles, puis décomposer à son tour cette ou ces unités dites de premier niveau, et ainsi de suite. La structure apparaît donc à travers les relations de niveau 
qu'entretiennent entre elles les unités, sous forme d'une hiérarchie.

Mais naturellement, cette démarche utile et méthodique ne se pose à aucun moment le problème de sa pertinence du point de vue psychologique. Les unités définies par le découpage sémiologique sont-elles perçues comme unités par l'auditeur ? Et surtout, des unités définies à un niveau déterminé, occupant une place particulière dans la hiérarchie structurale, sont-elles perçues fonctionnellement à ce niveau ou non ? D'une certaine manière, une unité de niveau III par exemple (supposant donc trois fois l'opération de segmentation) peut-elle être perçue par l'auditeur, qualitativement et psychologiquement, comme une unité de niveau supérieur, parce qu'ayant finalement une même prégnance dans le déroulement de l'oeuvre?

Nous pouvons donner une illustration de ce problème à partir de l'analyse du Prélude de Pelléas, tentée à plusieurs reprises, notamment par Ruwet (1972) puis par Nattiez (1975). La hiérarchie structurale qui se dégage, fait apparaître que les quatre premières mesures constituent une unité de deuxième niveau, tandis que les mesures 5 et 6 constituent une unité de quatrième niveau. Le critère adopté était alors (entre autres) la longueur des unités : dans un sondage auprès d'étudiants d'un séminaire de psychologie qui ne connaissaient pas le Prélude, nous avons obtenu un découpage auditif qui revenait à considérer au contraire ces deux unités de même niveau et de même structure : pour nos auditeurs, les quatre premières mesures se subdivisent en deux unités thématiques, les mesures 5 et 6 se subdivisent en deux unités également (mesure 5 d'une part, mesure 6 d'autre part), et la prégnance de ces subdivisions est rigoureusement la même, elles sont perçues avec la même intensité dans les deux cas. L'analyse dite de niveau neutre nous propose donc une organisation d'unités de longueurs différentes qui doit être croisée avec ce que l'analyse esthésique met en lumière : une alternance perceptive prégnante des unités longues et des unités deux fois plus brèves, comme si, pour l'auditeur, toute la partition était construite sur cette alternance. Or ce fait ne contredit pas l'organisation en différents niveaux que l'on trouve dans l'analyse du niveau neutre ; il permet au contraire de poser certaines questions fondamentales sur le style : cette tendance à percevoir équivalentes des unités courtes et rapides et des unités longues et plus lentes est-elle la conséquence d'une structure particulière que l'on retrouve dans d'autres oeuvres de Debussy ? Le style, d'une façon plus générale, réside-t-il dans une certaine façon qu'a le compositeur de faire 
entrendre à l'auditeur des réalités sonores originales à partir de structures générales et de procédés d'écriture inventoriés dans l'analyse du niveau neutre?

Bref, nous formulons l'hypothèse que la segmentation n'est pas seulement une opération logique de l'analyse sémiologique, mais aussi un processus perceptif réel à l'oeuvre au cours de l'audition, et qui permet au sujet de décoder la forme qui lui est soumise. Cette hypothèse peut être précisée dans trois directions :

a) Cette segmentation repose sur la perception de changements qualitatifs plus ou moins prégnants dans le flux temporel musical ;

b) Cette segmentation dépend de " modèles " de référence, codés et stockés en mémoire, qui ont été appris essentiellement par l'intermédiaire des processus d'acculturation ;

c) Cette segmentation dépend également de la structure " profonde " de l'oeuvre, et la dévoile en partie ; du moins, la façon dont s'opère la segmentation perceptive, le nombre d'unités qui en résultent et leur durée, ainsi que les contrastes, les ruptures ou les éléments dynamiques qui les délimitent, engendrent des comportements sémantiques (pour peu qu'on les déclenche par une demande explicite adressée au sujet) qui témoignent de la façon dont le style est décodé par le sujet à travers cette expérience particulière, la façon dont est signifiée une organisation particulière du temps musical.

\section{B - Perception du changement et segmentation}

La notion de changement est essentiellement temporelle et structurale : elle implique la perception d'une relation entre l'« avant " et l'« après ", donc la perception d'une succession contrastive introduisant dans le flux continu du temps une rupture. Comme l'écrit Fraisse, "le changement discontinu, pour être perçu, implique que je perçoive non seulement l'état $A$ puis l'état $\mathrm{B}$, mais le passage de l'un à l'autre, c'est-à-dire leur succession " (1957: 70). C'est donc le passage qui constitue la réalité perceptive de la relation, base des structures musicales. $\mathrm{Du}$ moins, toute pièce musicale s'organise-t-elle à l'audition en " parties " plus ou moins longues, repérées par le fait que chacune d'elles présente, par rapport à celle qui la précède, une qualité différente, et que, d'une certaine manière, le changement de partie introduit une discontinuité entre des ensembles sonores délimités qui, chacun pour eux, "réalisent une forme de synthèse presque statique du devenir " (Fraisse $1957: 97$ ). 
On peut donc penser que la netteté plus ou moins grande de ces parties, l'aisance avec laquelle les contrastes, les ruptures, les entrées de thèmes sont perçus, la prégnance de tel ou tel élément par rapport aux autres, constituent une hiérarchie plus ou moins forte d'unités s'emboîtant les unes dans les autres ou se juxtaposant, qui témoigne de l'organisation intrinsèque de la pièce musicale, ou tout au moins, de la part de cette organisation qui donne lieu à des comportements réactifs observables chez le sujet.

Ainsi le style apparaît d'abord comme cette hiérarchie perceptive de changements et des unités qu'ils délimitent, la force de cette hiérarchie dépendant du degré de différenciation et d'articulation des parties entre elles, et du nombre de différenciations effectivement perçues: une forte hiérarchie implique quelques changements très prégnants, quelques ruptures majeures, et, entre ces changements ou ruptures, d'autres changements moins prégnants, et, entre ces changements moins prégnants, des changements encore moins prégnants, et ainsi de suite, mais toujours en petit nombre à chaque niveau. De ce point de vue, une hiérarchie en arbre binaire serait une hiérarchie particulièrement forte ; une hiérarchie faible implique à l'inverse, soit de nombreux changements également prégnants et perçus d'emblée, avec entre eux des changements très faiblement perçus et en relativement grand nombre, soit des changements dont l'organisation est très mouvante, en ce sens qu'aucun contraste ou aucune rupture nette n'apparaît.

C - L'exemple de la comparaison de la Puerta del Vino avec un Intermezzo de Brahms.

Cette première hypothèse revient à considérer la segmentation d'une pièce musicale comme un processus de décodage que l'opération correspondante en analyse sémiologique ne reflète qu'en partie. L'étude comparée de la structure perceptive d'un autre des vingt-quatre Préludes, La Puerta del Vino, avec l'Intermezzo en mi bémol mineur op. $118 n^{\circ} 6$ de Brahms, montre comme la description correcte des hiérarchies de changements permet de poser des problèmes de fond concernant le style.

Nous rappellerons donc ici les principaux résultats de ces expériences (Imberty 1977 et 1981) afin de pouvoir les confronter ensuite à ceux obtenus avec la Cathédrale engloutie. La procédure expérimentale était la même que celle qui sera décrite ci-dessous, et comportait notamment deux auditions expérimentales, après une audition de familiarisation avec la pièce. 


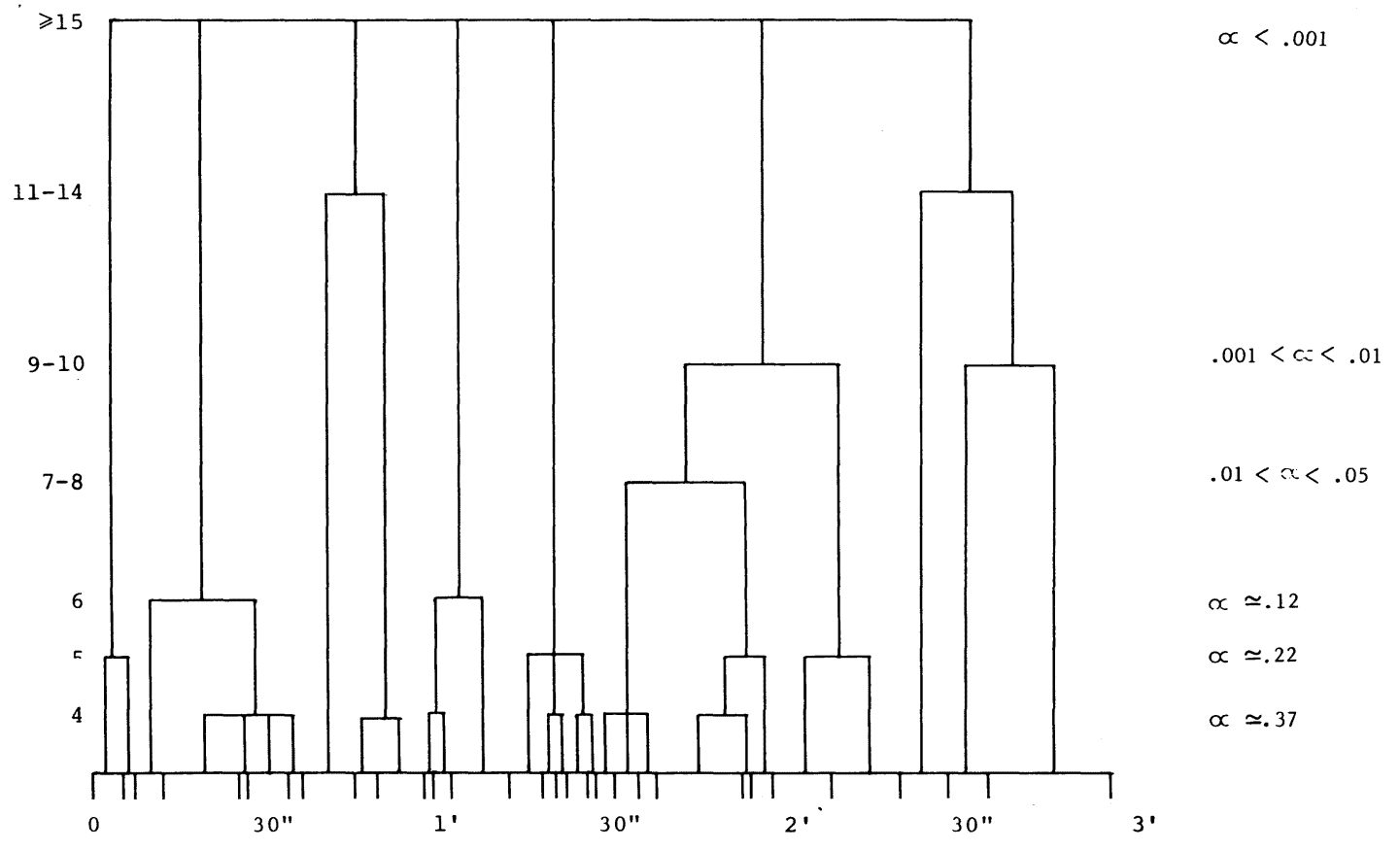

Figure 1

Structure perceptive de la Puerta del Vino de Debussy 


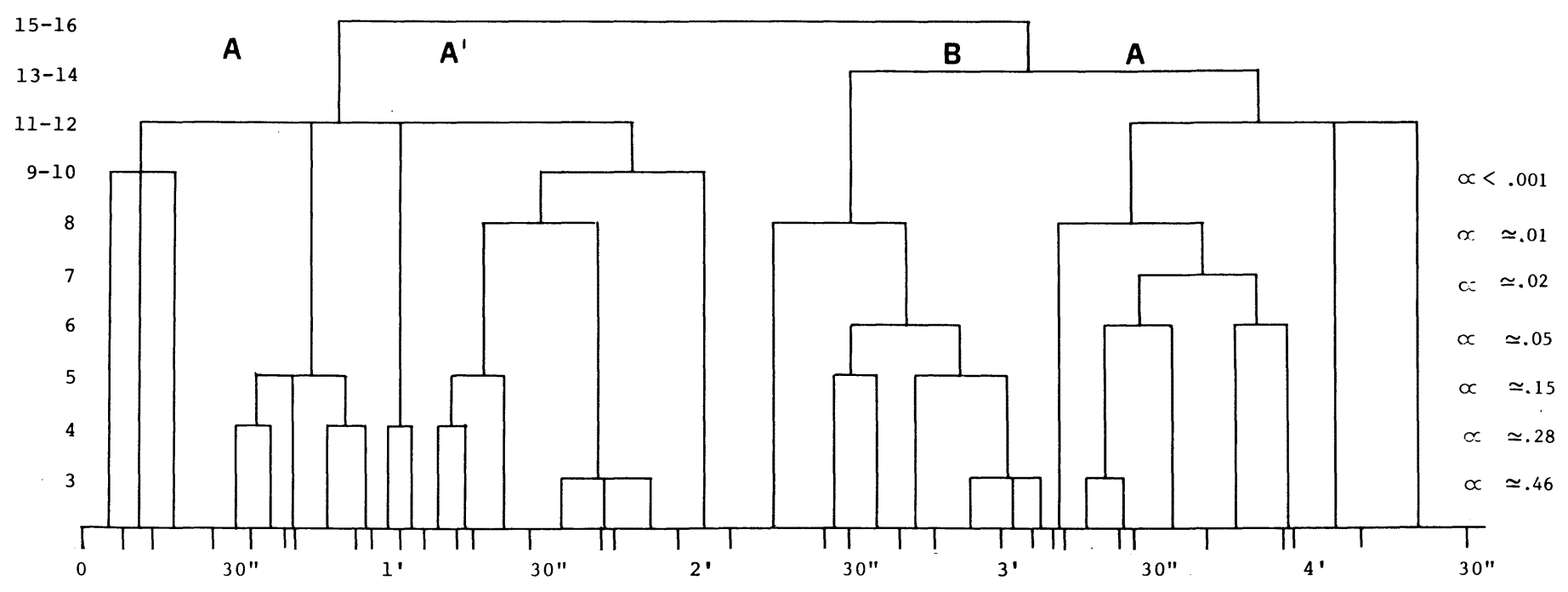

Figure 2

Structure perceptive de l'Intermezzo de Brahms 
Les figures 1 et 2 représentent les hiérarchies obtenues en sommant les réponses de deux auditions expérimentales. Ces hirarchies sont construites ainsi : chaque unité de la base de l'arbre est bornée par deux changements perçus par les sujets, et dont la fréquence de perception indique la prégnance. Les niveaux d'embranchements sont donc définis par ces fréquences, ou plus exactement par les seuils de significativité statistique correspondant, et les valeurs critique de la table de la loi de Poisson. Ainsi, dans la figure 1 (structure perceptive de La Puerta del Vino), les deux premières unités sont séparées par un changement indiqué par cinq sujets (total des deux auditions, seuil $P=.22$ ), tandis que la deuxième et la troisième sont séparées par un changement perçu par quinze sujets (et plus) (seuil $\mathrm{P}=.001$ ).

D'après ces figures, on conçoit fort bien comment la perception d'une oeuvre musicale est une organisation hiérarchique de changements, contrastes et ruptures perçus au cour de l'audition. La comparaison entre les deux figures montre immédiatement que cette organisation est extrêmement différente d'une pièce à l'autre : l'Intermezzo est plus fortement hiérarchisé que la Puerta del Vino, ce qui indique combien les unités s'emboîtent les unes dans les autres, combien finalement les liens entre les diverses unités de la base de l'arbre sont forts, et les changements et les passages d'un état à un autre peu prégnants. Les unités sont ellesmêmes de durée plus longue. Chez Debussy au contraire, ces unités de la base sont d'emblée plus courtes et plus nombreuses, les passages d'une unité à l'autre sont plus nets et plus aisément perçus : la structure est de ce fait peu hiérarchisée, les éléments ne sont pas emboîtés mais juxtaposés. Au total, cette comparaison montre que l'organisation temporelle de l'oeuvre de Debussy correspond à un temps musical morcelé, fait d'instants juxtaposés, isolés les uns des autres, dont les liens apparaissent lâches aux sujets auditeurs. L'oeuvre procède par contrastes successifs et violents. Chez Brahms au contraire, le temps musical est unifié et continu, fait de coordinations constantes, de variations progressives, de lentes évolutions du devenir.

Plusieurs indices statistiques le montrent. Tout d'abord, le nombre de changements perçus au total, dès la première audition, est de 69 pour une pièce d'une durée de 175 secondes dans le cas de Debussy, de 77 pour une pièce d'une durée de 271 secondes dans le cas de Brahms. La densité des changements perçus est donc doublée quand on passe de Brahms à Debussy. D'autre part, les changements les mieux perçus (ceux qui correspondent au seuil de 
$P \leq .01$ sur les figures) dans chacune des pièces sont cependant plus prégnants chez Debussy que chez Brahms (les fréquences sont plus élevées). On note également (Imberty 1977 et 1981) que la réorganisation, d'une audition à l'autre, est plus importante chez Brahms que chez Debussy, comme si la structure de la Puerta s'imposait d'emblée, alors que la structure de l'Intermezzo est plus fuyante, favorisant les recentrations perceptives et laissant l'auditeur dans une relative incertitude quant à l'organisation sous-jacente. Enfin, si visuellement, la différence d'organisation des arbres hiérarchiques apparaît clairement aux figures 1 et 2, il est possible de tester cette différence en comptant les distances qui séparent les unités de la base au sommet de l'arbre : on appelle distance le nombre d'embranchements entre l'unité perceptive à la base et le sommet de l'arbre. Il est clair que, plus les distances sont grandes, plus la hiérarchie est forte, c'est-à-dire plus les unités de la base de l'arbre sont faiblement perçues et fortement emboîtées dans des unités supérieures plus vastes et plus organisées ; plus au contraire ces distances sont faibles, plus la hiérarchie est faible, les unités de base étant perceptivement très prégnantes, isolées les unes des autres, juxtaposées. On a vingt-huit unités pour la base de la Puerta del Vino et trente-deux unités pour la base de l'Intermezzo. Mais le tableau 1 montre que les distances sont très différentes, beaucoup plus fortes pour Brahms que pour Debussy $\left(X_{4}^{2}=11,4\right.$ significatif à $\left.\mathrm{P} \simeq .02\right) \cdot{ }^{1}$

\begin{tabular}{|c|c|c|}
\hline & $\begin{array}{c}\text { Nombre d'unités de la } \\
\text { base (Debussy) }\end{array}$ & $\begin{array}{c}\text { Nombre d'unités de la } \\
\text { base (Brahms) }\end{array}$ \\
\hline 2 & 7 & 0 \\
\hline 3 & 15 & 11 \\
\hline Distance 4 & 4 & 7 \\
\hline 5 & 2 & 10 \\
\hline \multirow[t]{2}{*}{6} & 0 & 4 \\
\hline & 28 & $\chi_{4}^{2}=11,44(.02)$ \\
\hline
\end{tabular}

Tableau I

Comparaison de la hiérarchisation des changements perçus chez Debussy et chez Brahms. On lit, à la ligne 1 et à la colonne 1 : dans la pièce de Debussy, 7 unités de la base sont à distance 2 du sommet ; colonne 2, aucune chez Brahms, etc. 
D - Modèles culturels et perceptifs de segmentation : le concept de macro-structure

Cette première analyse reste cependant incomplète. Dans une série d'expériences portant sur les conduites perceptives de reconnaissance de thèmes ou de parties d'une fugue ou d'une sonate, Francès (1958) montre que des sujets musiciens, connaissant ces formes, ont nettement plus de facilité que les sujets non musiciens pour identifier l'unité thématique. Mais il montre aussi que cette plus grande facilité provient non seulement d'une capacité de différenciation auditive plus fine, mais aussi d'un savoir qui fonctionne comme " référence " de ce qui est entendu. Les sujets musiciens ont généralement acquis la connaissance de cadres formels qui guident naturellement leur perception. Ce fait semble très général, et les études déjà nombreuses sur l'acculturation tonale ont largement montré que, même par simple fréquentation des oeuvres, des habitudes auditives se créent même chez les sujets les moins doués, et qu'elles déterminent pour une large part la structuration perceptive des stimuli.

On peut donc penser que la connaissance - implicite ou non - de certaines formes musicales très générales favorise certaines stratégies dans la segmentation auditive de la pièce musicale : telles sont la sonate par exemple, ou le rondo avec son refrain, ou toutes les formes classiques largement répandues dans la consommation musicale occidentale. Les formes tripartites notamment font partie des acquis culturels qui conditionnent nos habitudes auditives : il est aisé de concevoir qu'au moins pour un public amateur, a priori, toute oeuvre musicale comporte quelque chose comme une exposition, un développement, une réexposition, ou une idée $A$, une seconde idée B, puis un retour à A. Sans aller jusqu'à rejoindre Lévi-Strauss (1972) lorsqu'il affirme que toute musique est structurée comme une "aventure " (problèmetension, crise, dénouement-résolution), on voudrait au moins suggérer que cette structure, entretenue par trois siècles de musique tonale, et qui fut imposée par la syntaxe tonale ellemême, n'est peut-être pas éloignée des structures des récits qui s'organisent eux-mêmes selon un schéma assez voisin. Comparaison sans doute banale en ce qu'elle a été cent fois faite. Mais elle devient pertinente à partir du moment où il est possible de montrer que la mémorisation, en même temps que la segmentation de la pièce musicale, se fait en accord ou en conflit avec ce modèle acquis et renforcé par la culture commune aux sujets occidentaux. Â ce titre, le style ne serait plus seulement une hiérarchie perceptive de 
changements, plus ou moins forte selon les cas, mais en outre, une façon particulière de permettre cette hiérarchie au sein d'un modèle plus vaste et plus général transmis par la culture.

C'est pourquoi nous semble très suggestive l'étude de Kintsch et van Dijk (1975) à laquelle nous empruntons le concept de " macro-structure" : selon ces auteurs, tout discours est interprété, stocké en mémoire, rappelé en fonction d'une structure d'ensemble qu'ils appellent précisément sa macro-structure, et qui appartient au système des connaissances générales du sujet. Par une série d'expériences de rappel et de rappel résumé de textes, les auteurs dégagent cette macro-structure qu'ils décrivent comme une sorte de schéma général comprenant certaines cases vides, situées à des niveaux différents définis par une hiérarchie qui constitue cette macro-structure. Ces cases vides sont remplies par les informations spécifiques du texte. Le rappel du récit consiste alors en une réactivation de la macro-structure, système de décodage-encodage vide, qui appelle des informations spécifiques stockées en mémoire. La macro-structure apparaît donc comme une mise en ordre du temps du récit, elle permet d'en reconstituer les épisodes successifs selon des règles très générales issues des mécanismes cognitifs sous-jacents.

En ce sens, le concept de macro-structure peut nous être utile : schéma de structuration du temps, la macro-structure est, pour la musique, mise en ordre a priori d'évènements sonores, selon des règles issues des mécanismes perceptifs, qui permettent le repérage des changements dans le continuum sonore. En somme, la micro-structure d'une pièce musicale est constituée par la perception et la rétention mnémonique de quelques changements particulièrement prégnants, déterminant pour l'auditeur la progression globale de la pièce. Ces changements peuvent être imputables soit à un stéréotype culturel, soit - et dans ce cas, ils contredisent le stéréotype - à un style.

Quelques indications peuvent être données par une expérience complémentaire portant sur les deux pièces que nous avons analysées précédemment. Nous avons demandé (Imberty 1981) à des sujets n'ayant pas participé à la première expérience d'écouter chaque pièce, puis nous leur avons demandé de dire combien de parties ils avaient entendues et d'essayer de les décrire brièvement. Il s'agissait donc un peu d'une épreuve de mémoire en "rappel immédiat ". Or, si pour la pièce de Brahms, le stéréotype ABA sur lequel elle est manifestement construite, est immédiatement apparu dans les réponses de tous les sujets, musiciens et non 
musiciens, en revanche, pour la pièce de Debussy, les sujets non musiciens ont massivement indiqué également trois parties, selon un schéma dynamique symétrique ( "ça commence lentement et doucement, puis ça devient très fort et brutal, puis de nouveau lent et doux " ), la plupart d'entre eux allant jusqu'à oublier l'attaque ff des premiers accords. En l'absence d'un schéma structural simple, les sujets non musiciens ont donc simplifié à l'extrême pour ramener la pièce au même schéma que la pièce de Brahms, c'est-àdire à un schéma-type qu'ils connaissent par acculturation et qui oriente a priori le décodage de la structure.

\section{$\mathrm{E}$ - Les schèmes de la perception temporelle}

En dehors de ce système culturel de décodage, la macrostructure d'une pièce musicale suppose la mise en oeuvre de deux types de schèmes perceptifs temporels : des schèmes d'ordre, et des schèmes de relation d'ordre.

On peut les distinguer ainsi: les schèmes d'ordre ne concernent que les simples successions et les juxtapositions, au plan de la dynamique ; ils s'étendent donc à la fois aux relations proches et aux relations lointaines (croissance, diminution, répétition, imitation, accélération, ralentissement, etc.). Les schèmes de relation d'ordre concernent les relations organiques qui permettent d'établir des rapports entre les parties temporelles proches (thèmes, variations de thèmes, relations syntaxiques générales, bref, tout ce qui tend à synthétiser le temps dans une sorte de présent psychologique saisi en tant que tel). Si l'on veut, les schèmes d'ordre assimilent le temps en tant que devenir et écoulement successif irréversible ; les schèmes de relation d'ordre assimilent le contenu en évènements sonores variés de cet écoulement, pour le saisir en dehors du successif dans une cohérence plus abstraite.

Ce sont les schèmes de relation d'ordre qui sont principalement à l'oeuvre dans la perception des structures fortement hiérarchisées (cf. Brahms, figure 2), où certains changements apparaissent plus comme des enchaînements ou de transitions à fonction coordinatrice que comme des contrastes ou ruptures dans le devenir temporel. Ces changements sont, la plupart du temps, peu prégnants et se situent aux niveaux inférieurs de l'arbre. Mais il est clair aussi que les stéréotypes culturels réalisent des relations d'ordre qui synthétisent la forme sonore en dehors de son irréversibilité immédate. En effet, la répétition de l'audition, dans la mesure où elle favorise un certain apprentissage, déclenchent 
des anticipations perceptives qui lient les évènements successifs à travers des relations fortes : or nous avons constaté (Imberty 1981) que cet apprentissage n'est efficace que pour la pièce de Brahms, construite sur le stéréotype ABA, et non pour celle de Debussy. La connaissance implicite du stéréotype favorise donc l'assimilation de la forme aux schèmes de relation d'ordre, mais inversement, plus la forme est hiérarchisée et coordonnée selon des règles strictes, plus la perception se fait par assimilation aux schèmes de relation d'ordre.

Au contraire, une faible hiérarchie suppose principalement la mise en oeuvre des schèmes d'ordre, et les ruptures brutales et nombreuses (Debussy, figure 1) consacrent les déplacements de l'équilibre perceptif qui morcellent le devenir musical. En particulier, l'absence de stéréotype formel classique qui aurait pu servir de repère aux sujets, dans la mesure où il correspond à une connaissance a priori de ce qu'est une pièce musicale en culture occidentale, a rendu impossible tout apprentissage au cours des auditions successives, laissant finalement une impression d'incoordination, voire d'incohérence. Bref, les schèmes d'ordre orientent l'attente perceptive vers un événement qui demeure imprécis et vague, les schèmes de relation d'ordre au contraire assimilent la structure sonore à une thématique déterminée qui ne se comprend qu'au-delà du déroulement temporel immédiat.

Un point illustre particulièrement ces hypothèses générales : il s'agit de ce qu'on peut appeler les points de condensation perceptive de la macro-structure. Il y a en effet un moment privilégié où, brusquement, la totalité de la pièce musicale prend un sens, c'est-à-dire un moment, au cours de l'audition, où l'auditeur, de manière inconsciente ou implicite, pressent le schéma temporel général, la direction essentielle, le but vers lequel le compositeur le conduit. C'est ce moment qui conditionne la perception de tous les évènements sonores qui vont suivre, mais, rétroactivement, tisse un lien avec ceux qui viennent de s'écouler. Notamment, on peut dire que le point de condensation maximum oriente la perception, c'est-à-dire déclenche les schèmes de relation d'ordre qui vont engendrer les exigences de retour et de symétrie : si le point de condensation maximum est en particulier situé au sommet d'intensité de la pièce, au climax, le sujet attend normalement une chute de cette intensité, en même temps qu'un dénouement de la tension. Si le point de condensation maximum est situé à l'amorce d'un nouveau thème, donc après une rupture ou un contraste, le sujet s'attend, par le fait même qu'il quitte un certain climat, à y 
revenir. Toute la partie antérieure prend son sens de but à atteindre et à retrouver, par le fait même qu'on la quitte. On constate que les deux points de condensation maximum pour la pièce de Brahms coïncident, le premier à 2'07' (mesure 41) à la rupture centrale, amorce du nouveau thème et de la nouvelle partie, le second à 3'10" (mesure 61-62) à l'attente du retour prévisible de la partie initiale (niveaux supérieurs des embranchements, figure 2).

La macro-structure est donc bien le résultat de coordinations de schèmes perceptifs temporels qui assimilent la réalité perçue (anticipations), tout en s'accommodant aux particularités stylistiques de la pièce, et en réorganisant le devenir à partir de points privilégiés de condensation perceptive.

Mais il est évident que la macro-structure dépend des stéréotypes culturels dont on vient de voir l'importance, et que les sujets ont appris par acculturation. En particulier, la musique de Brahms, tout comme l'ensemble de la musique tonale, repose sur ce temps réversible et symétrique qu'impose la syntaxe (enchaînements tonique - dominante - tonique, etc.). Il est évident que la nécessité d'un retour après un "détour ", d'un "dénouement " après une " crise ", pour employer les termes de Lévi-Strauss dans l'Homme $\mathrm{Nu}$ (1972 : 589-590), n'a été acquise par la musique occidentale que relativement tard. Et il apparaît que les schèmes de relation d'ordre ne fonctionnent qu'en assimilant une expérience du temps par ailleurs largement culturalisée.

Or de ce point de vue, la pièce de Debussy apparaît très différente : la macro-structure faiblement hiérarchisée correspond au fait que les schèmes d'ordre, en recentrant la perception à chaque changement perçu, séparent les zones temporelles et renforcent le poids psychologique des segmentations. On remarque alors (figure 1) qu'il n'y a pas, chez Debussy, de point de condensation maximum unique ou en nombre restreint correspondant à ceux d'un stéréotype. Au niveau supérieur de l'arbre, il y a fragmentation de la structure en plusieurs points équivalents. Il n'y a donc pas de point d'où l'évolution dynamique apparaît au sujet, et prend un sens d'unité coordonnée, maîtrisable conceptuellement hors du temps. En outre, les points de condensation les plus forts se trouvent au début, comme si l'essentiel de l'oeuvre se trouvait résumé dans une opposition fondamentale donnée tout de suite, et qui n'évoluera pas.

Il faut cependant nuancer : en fait, toute perception musicale met probablement en jeu à la fois les schèmes d'ordre et les 
schèmes de relation d'ordre. Il arrive seulement que l'un des deux systèmes domine l'autre. À cet égard, les pièces analysées sont encore exemplaires : lorsqu'il y a coïncidence entre l'organisation thématique (qui relève au plan perceptif des schèmes de relation d'ordre) et l'organisation dynamique (qui relève des schèmes d'ordre), les schèmes de relation d'ordre assimilent l'ensemble de la structure thématique, et au besoin renforcent la hiérarchisation par l'intervention des' stéréotypes qui, depuis deux siècles, supposent cette coïncidence. C'est le cas pour la pièce de Brahms, dont la macro-structure est entièrement construite sur la thématique. Lorsqu'au contraire, il y a décalage entre l'organisation thématique et l'organisation dynamique, les schèmes d'ordre assimilent l'ensemble de la structure aux progressions, diminutions, croissances, ruptures, et la thématique est reléguée au second plan : la figure 2 montre nettement que ce qui sert de " réexposition " dans la Puerta del Vino n'est absolument pas perçu comme tel par les sujets (1'57", embranchement peu significatif statistiquement $P=.22$ ). L'oeuvre musicale, dont la structure est alors peu hiérarchisée, est alors représentation d'images temporelles brutes, liées directement aux expériences individuelles ou collectives du temps existentiel.

On peut donc penser de cet ensemble de résultats, que les modes de décodage renvoient d'une certaine façon à la structure compositionnelle elle-même, qu'ils permettent au chercheur d'établir des liens théoriques forts entre l'esthésique et le poïétique, et que par conséquent, ils nous renseignent sur l'une des fonctions essentielles de la musique pour l'homme : la représentation et la maîtrise symboliques du temps, clé d'une sémantique psychologique de la musique.

\section{Présentation de l'expérience sur la Cathédrale engloutie}

\section{A - Les objectifs de l'expérience.}

Que le lecteur ne s'impatiente pas! Ce long détour par l'analyse d'une pièce de Brahms (comparée tout de même à la Puerta del Vino) était nécessaire pour faire mieux comprendre le point de vue adopté à propos de la Cathédrale engloutie. En effet, nous pouvons maintenant poser un certain nombre de questions précises.

La première concerne évidemment la faiblesse de la hiérarchie perceptive établie pour la Puerta: se retrouve-t-elle dans la Cathédrale, et si oui, constitue-t-elle un aspect majeur du style de 
Debussy, comparé à celui de Brahms (et - peut-être plus généralement - à l'écriture romantique) ?

La seconde question concerne la macro-structure, tant du point de vue d'un éventuel impact de stéréotypes formels usuels, que du point de vue de la distribution des points de condensation perceptive. Là encore, ce qui différencie la Puerta del Vino de l'Intermezzo de Brahms, est-il un des aspects du style de Debussy et de l'organisation du temps musical dans son oeuvre?

Enfin, ultérieurement, une troisième question pourra être abordée, celle qui, à dire vrai, constituait l'objet initial de cette étude : quel impact la structure perceptive de la pièce a-t-elle sur l'organisation des significations associées au cours ou après l'audition ? La Puerta del Vino, comme l'Intermezzo, ont donné lieu aussi à cette étude (Imberty 1978 et 1981) qui pourra donc être menée comparativement à propos de la Cathédrale.

\section{B - Déroulement de l'expérience}

Trente sujets participent à l'expérience. Tous sont adultes, la plupart étudiants, et tous s'intéressent à la musique. Leur niveau d'éducation dans ce domaine est celui d' "amateurs ", mais ils n'ont, la plupart du temps, aucune éducation technique.

L'expérience se déroule individuellement, et l'expérimentateur lit au sujet la consigne suivante : "Une oeuvre musicale est composée de parties plus ou moins longues qui sont perceptibles à l'audition par l'intervention, dans le flux musical, de changements d'atmosphère, de rythme, d'harmonie, ou par l'apparition de thèmes. Certains de ces changements, contrastes ou ruptures sont immédiatement repérables, et ce sont eux qui délimitent les parties principales. D'autres sont plus ténus, et délimitent, à l'intérieur des grandes parties, des unités plus petites. On peut présenter les choses autrement en faisant une comparaison : un poème est composé de strophes, les strophes sont elles-mêmes composées de vers. Mais à l'intérieur de la strophe, les vers peuvent aussi se regrouper par deux. On a donc ainsi une séries d'unités ou parties qui s'emboîtent les unes dans les autres, des plus grandes aux plus petites. Votre tâche va consister à indiquer à votre expérimentateur le moment où vous percevrez des changements (contrastes, thèmes, atmosphères différentes, etc.) au cours de l'audition d'une courte pièce pour piano. " On indique alors au sujet le moyen de faire connaître sa réponse à l'expérimentateur (signe convenu), puis on lui explique qu'il va d'abord entendre la pièce une première fois pour se familiariser avec elle, sans qu'il ait à donner de 
réponses. Ensuite, on lui demande d'indiquer, au cours d'une première audition expérimentale les coupures ou changements les plus nets ; au cours d'une seconde audition expérimentale, on lui demande alors d'indiquer tous les changements qu'il perçoit ( $y$ compris ceux signalés lors de la première audition).

L'expérimentateur dispose d'une feuille préparée à l'avance, graduée en indications de temps (en secondes), et d'un chronomètre. Il note sur la feuille de protocole les temps où le sujet perçoit un changement.

\section{C - Dépouillement des résultats.}

Le problème de procédure de dépouillement tient en ceci : tous les sujets n'ont pas le même temps de réaction, et par conséquent, certains sujets ont probablement tendance à donner leurs réponses en retard sur le changement perçu. Si cet inconvénient pouvait être limité en ce qui concerne l'expérimentateur, entraîné au préalable, il était inévitable en ce qui concerne les sujets. Les résultats montrent dans cette expérience, comme dans les précédentes, que les réponses les plus fréquentes sont souvent localisées à une seconde près. Dans la plupart des cas, l'analyse de la partition permet de dire s'il s'agit d'un retard dû au temps de réaction des sujets, ou s'il s'agit de la perception d'une réalité sonore distincte. Il est même probable que le tempo relativement lent de la Cathédrale a entraîné des retards plus nombreux que pour la Puerta del Vino : le temps de réaction à un signal est en effect directement lié à la durée effective de ce signal. Or la durée moyenne des signaux est ici directement dépendante du tempo de la pièce. C'est le cas, par exemple, à la mesure 13 (figure 13 à 50"-52"), où le changement perçu est lié au retour du registre d'extrême grave, lequel dure en fait les deux derniers temps de la mesure. Mais il est clair que, lorsqu'on s'intéresse aux changements moins importants ou moins prégnants, indiqués par trois ou quatre sujets seulement; il devient beaucoup plus difficile de dire si la réponse correspond à une erreur due au temps de réaction du sujet, et concerne un changement perçu une ou deux secondes avant, ou s'il s'agit d'un changement réellement perçu : à la limite, dans une pièce comme la Cathédrale engloutie, compte tenu de son tempo, on peut imaginer que toute note perçue soit perçue comme changement. D'ailleurs, sur une durée de 330 secondes, dès la première audition, on obtient 226 changements localisés distinctement. Le phénomène était déjà net pour la Puerta del Vino. 


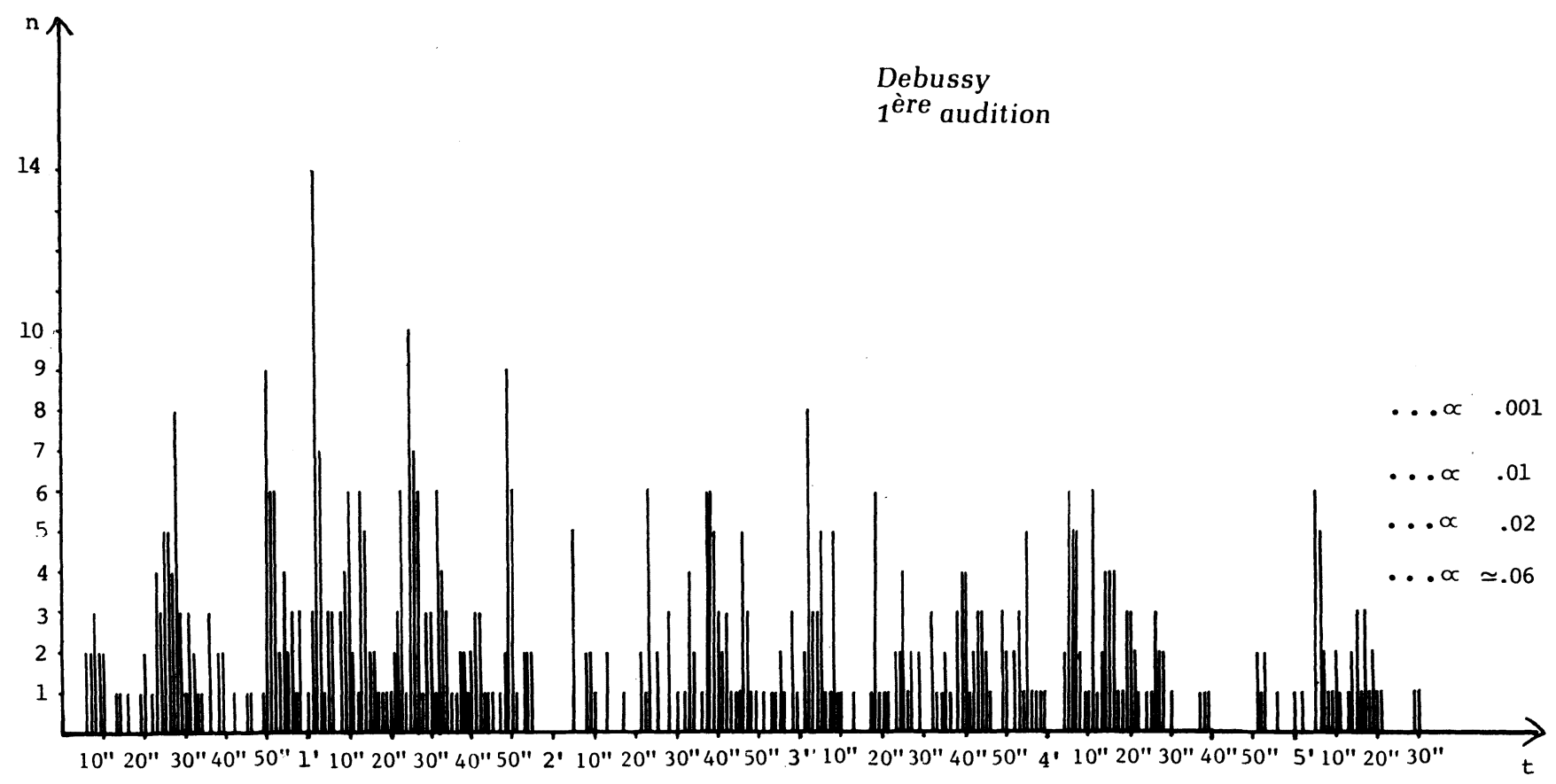

Figure 3 


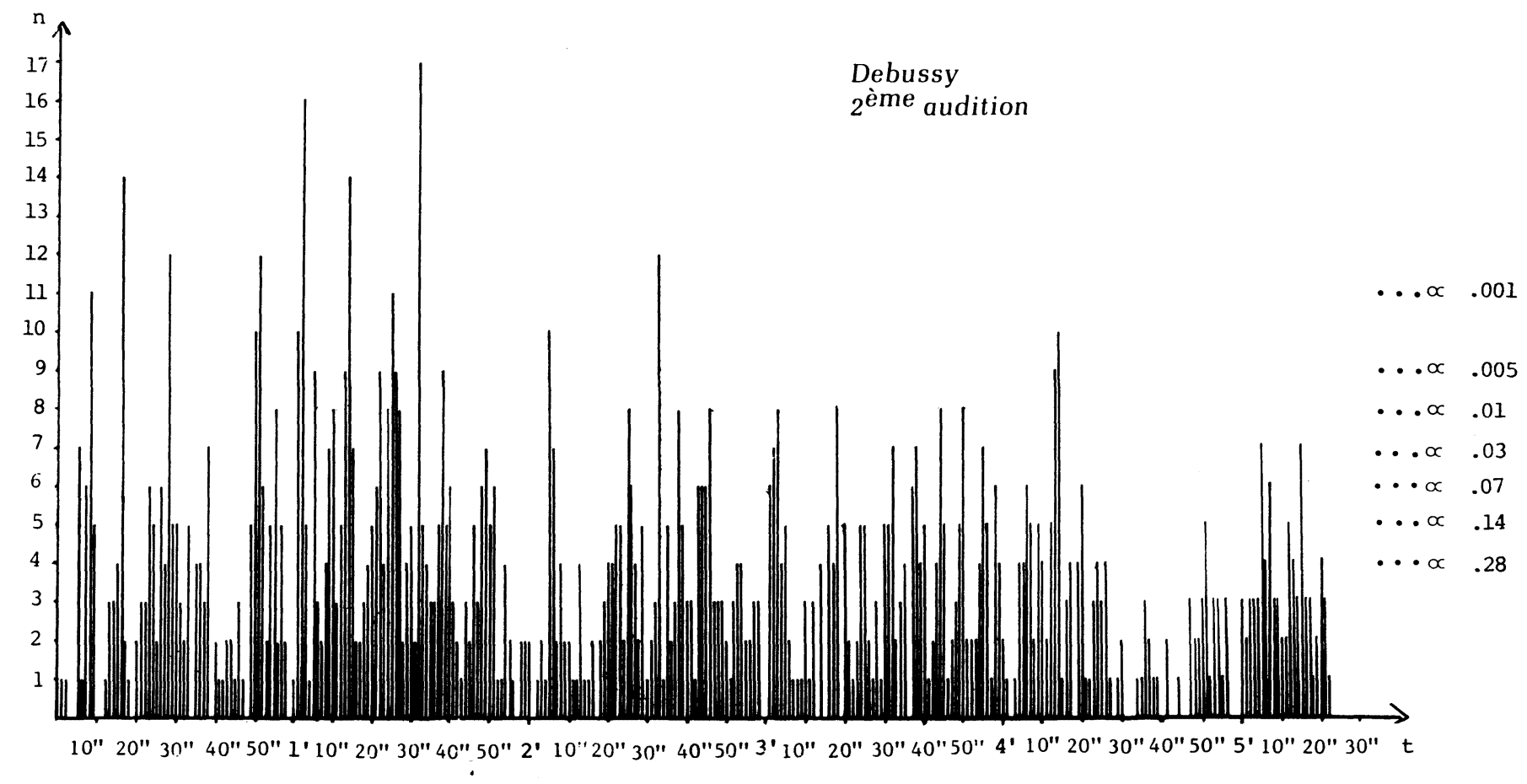

Figure 4 


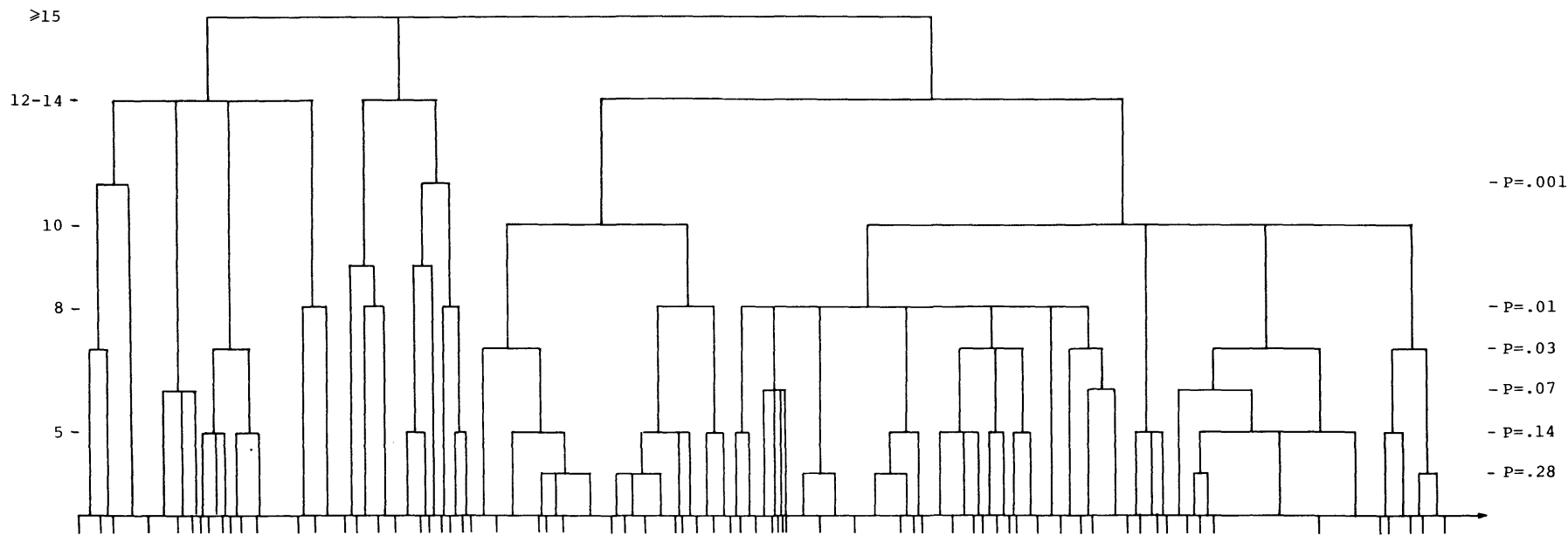

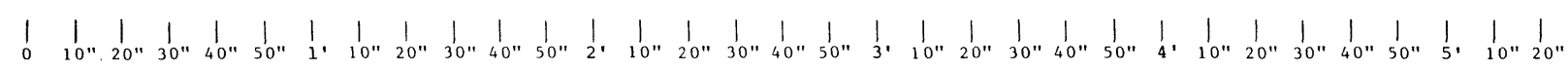

Figure 5

Structure perceptive de la Cathédrale engloutie 
Le problème était donc de savoir s'il fallait regrouper certaines réponses et les localiser au même endroit sur la partition. Sur la figure 3 , doit-on considérer que les réponses données à la $50^{\mathrm{e}}$, à la $51^{\mathrm{e}}$ et à $\mathrm{la} 52^{\mathrm{e}}$ secondes concernent la perception du même changement ? Si, dans ce cas, la réponse parait évidente, il n'en est pas toujours ainsi. Par conséquent, et par prudence, nous n'avons effectué aucun regroupement de réponses dans l'interprétation des résultats : on objectera qu'on perd ainsi une part de l'information. Toutefois, en ce qui concerne les changements les plus fréquemment perçus (significativement, jusqu'à un seil de $\mathrm{P} N$.10), cette façon de procéder ne change en rien l'identification des éléments déterminants sur la partition. Ce qui est d'autant plus facile à comprendre que la distribution des fréquences des changements perçus est très dissymétrique.

En effet, l'inconvénient que nous venons de signaler se trouve neutralisé en grande partie par le fait que cette distribution des fréquences des changements perçus est poissonnienne. Il est aisé de déterminer, pour chacune des figures obtenues, la valeur critique au-delà de laquelle on peut considérer que la fréquence est significative; on s'aperçoit alors que les changements pour lesquels il pourrait y avoir ambiguité ne sont pas significativement perçus. On trouvera ces indications matérialisées sur les figures 3 et 4 .

\section{La macro-structure de la Cathédrale engloutie.}

\section{A - Les résultats globaux.}

Les comparaisons statistiques avec l'Intermezzo et la Puerta del Vino seront difficiles, car le nombre de sujets participant à l'expérience présente n'était pas le même que pour les expériences précédentes : 30 ici au lieu de 20 antérieurement. Or l'augmentation du nombre de sujets, si elle donne une plus grande fiabilité aux conclusions qu'on peut tirer des résultats globaux, a cependant une conséquence directe sur l'appréciation du nombre de changements réellement perçus : en effet, les changements perçus par un seul sujet peuvent être parfois l'indication d'un changement situé antérieurement, mais signalé trop tard. Or le risque qu'il en soit ainsi et qu'il y ait ambiguïté au niveau du dépouillement augmente avec le nombre de sujets, si bien que les phènomènes de retard dûs aux temps de réaction des sujets s'additionnent et gonflent quelque peu le nombre de localisations différentes des changements perçus au cours de l'audition. On peut cependant 
assez bien se rendre compte de la tendance générale, qui est que la structure perceptive de la Cathédrale engloutie apparaît du même type que celle de la Puerta del Vino, à la fois par le nombre et la densité des changements perçus, par la multiplicité des points de condensation perceptive, enfin par la dissymétrie globale de la structure, plus morcelée au début qu'à la fin (cf. figures 3 et 5).

a) Le nombre de changements perçus : sous réserve de ce qui vient d'être dit concernant le nombre de sujets de l'expérience, il apparaît que, dès la première audition expérimentale, le nombre de changements perçus est très élevés par rapport à la durée totale de la pièce : 226 changements localisés pour une durée de 330 secondes, alors que pour Brahms la proportion était exactement inverse (77 changements localisés pour une durée de 271 secondes). La différence est encore plus marquée à la seconde audition.

On pourrait tenter une comparaison encore plus précise entre les trois pièces en ramenant le nombre de changement perçus et localisés dans la Cathédrale à ce qu'il aurait pu être avec seulement 20 sujets (nombre de sujets des expériences précédentes) par une simple règle de trois. Le tableau II propose cette comparaison sur les nombres " corrigés " pour la Cathédrale, tout en rappelant les nombres bruts. On lit ce tableau ainsi : le chronométrage ayant été réalisé à la seconde, chaque pièce est définie comme une suite de $\mathrm{N}$ moments séparés par une seconde d'intervalle. Le nombre de changements perçus peut donc être considéré comme le nombre de moments où il y a eu déclenchement d'une réponse perceptive chez au moins un sujet : soit n ce nombre. La différence $\mathrm{N}$ - n représente alors le nombre de moments où il n'y a eu aucune réaction d'aucun sujet. On peut alors effectuer la comparaison entre le nombre de moments qui ont déclenché une réponse au moins (nombre de changements perçus) dans chaque pièce, et le nombre de moments où il ne s'est rien passé. Cela revient à comparer la durée totale des changements perçus par rapport à la durée totale de la pièce. 


\begin{tabular}{|c|c|c|c|c|c|}
\hline & $\begin{array}{c}\text { Première } \\
\text { audition } \\
\text { Durée sans }\end{array}$ & $\begin{array}{c}\text { Deuxième } \\
\text { audition } \\
\text { changement }\end{array}$ & $\begin{array}{l}\text { Première } \\
\text { audition } \\
\text { Durée des }\end{array}$ & $\begin{array}{c}\text { Deuxième } \\
\text { audition } \\
\text { hangements }\end{array}$ & $\begin{array}{l}\text { Durée } \\
\text { totale }\end{array}$ \\
\hline $\begin{array}{l}\text { Cathédrale } \\
\text { (bruts) }\end{array}$ & 104 & 40 & 226 & 290 & 330 \\
\hline $\begin{array}{l}\text { Cathédrale } \\
\text { (corrigés) }\end{array}$ & 180 & 137 & 150 & 193 & \\
\hline Intermezzo & 194 & 143 & 77 & 128 & 271 \\
\hline la Puerta & 106 & 40 & 69 & 125 & 175 \\
\hline \multicolumn{6}{|c|}{$\begin{array}{l}\text { Valeurs des } \chi_{1}^{2}: \text { tous sont hautement significatifs dans les comparaisons } \\
\text { sur les nombres bruts. } \\
: \text { en nombres corrigés, sont significatifs les } \chi_{1}^{.2} \text { de } \\
\text { comparaison entre la Cathédrale et l'Intermezzo, aussi } \\
\text { bien pour la première que pour la seconde audition } \\
(\mathrm{P}=.01) \text {. } \\
: \text { en nombres corrigés, non significatif entre la Cathédrale } \\
\text { et la Puerta, à la première audition ; significatif à la } \\
\text { deuxième audition, mais c'est la Puerta qui paraît alors } \\
\text { avec la durée des changements la plus forte par rapport } \\
\text { à la durée totale, alors que c'est l'inverse en nombres } \\
\text { bruts. }\end{array}$} \\
\hline
\end{tabular}

Tableau II

Comparaison de la Cathédrale engloutie avec l'Intermezzo de Brahms et la Puerta del Vino (nombre de changements perçus).

Encore une fois, on prendra ces comparaisons avec quelque prudence. Mais que ce soit en nombres bruts ou en nombres corrigés, la comparaison de la Cathédrale avec l'Intermezzo est toujours très nette : la densité des changements est beaucoup plus élevée chez Debussy que chez Brahms, et ce résultat confirme celui trouvé avec la Puerta del Vino (Imberty 1977 et 1981). Ce résultat est d'autant plus intéressant qu'au moins apparemment, la pièce est très différente de la Puerta, les contrastes dynamiques moins marqués, les progressions d'intensité, voire les passages d'un épisode à l'autre, plus continus. Car, jusqu'à un certain point, on aurait pu imputer la très grande différence entre l'Intermezzo de Brahms et la Puerta à un artifice expérimental qui consistait à avoir précisément choisi ces deux pièces d'atmosphère très différente. À certains égards, la Cathédrale, notamment par son tempo et son étalement dans la durée, est plus proche de l'Intermezzo. Or la comparaison stylistique reste tout aussi nette, ou, plus exactement, la densité des changements perçus au cours 
de l'audition est bien un indice très fort du style de chaque compositeur. Le morcellement de la durée apparaît aussi net dans la Cathédrale que dans la Puerta, et c'est bien là un résultat de portée très générale, dont la signification a été exposée précédemment : le temps debussyste est un temps qui n'évolue pas, ne progresse pas, mais s'immobilise en mille instants chatoyants, juxtaposés en des images sonores multiples qui se succèdent sans lien rationnel tangible. Comme nous l'avions déjà suggéré ailleurs, la lenteur debussyste n'aboutit pas à une fusion du devenir musical, mais les sons étirés, les silences, séparent et disloquent, conduisant ainsi l'écriture musicale aux antipodes de ce qu'elle fut pendant un siècle de romantisme.

La différence concernant la Cathédrale et la Puerta est plus difficile à interprèter pour l'instant. L'analyse détaillée de la macro-structure de la Cathédrale permettra peut-être de comprendre de quoi il s'agit. Mais il faut dire encore une fois que cette différence ne doit être considérée qu'avec prudence : en nombres "corrigés", c'est la Puerta qui paraît avoir donné lieu à la perception du plus grand nombre de changements, alors que la différence s'inverse avec les nombres bruts. En fait, cette incertitude tend plutôt à montrer que, même s'il existe une différence dans l'organisation perceptive des deux pièces, celle-ci est moins nette que dans les comparaisons avec Brahms, et tient à d'autres paramètres que les paramètres de prégnance perceptive.

b) La multiplicité des points de condensation perceptive : on y reviendra dans l'analyse de la macro-structure, mais d'ores et déjà, de ce point du vue également, la Cathédrale est tout à fait comparable à la Puerta : six points de condensation perceptive (seuil à $P=.001$ ), dont l'espacement est très irrégulier, ceci pour la première audition. À la seconde audition, aucune réorganisation spectaculaire, comme on pouvait en observer chez Brahms, mais cinq points de condensation perceptive nouveaux apparaissent, sans qu'ils modifient l'aspect d'ensemble de la structure. Simplement, celle-ci apparaît encore plus morcelée qu'à la première audition, surtout au début de la pièce. À nouveau, comme pour la Puerta, l'impression que toute la matière de la pièce est contenue dans les deux premières pages de la partition, et qu'après la retombée des grands accords, plus rien ne se passe.

c) La dissymétrie de la structure perceptive : ce dernier point est très lié au précédent. On vient de le voir (et les figures 3 et 4 sont à cet égard très parlantes), les points de condensation perceptive principaux sont massés au début. Ensuite, tout se passe comme si 
les ruptures et les changements étaient atténués, comme si la macro-structure apparaissait moins chaotique, plus ordonnée et maîtrisée. Ce constat se retrouve pour la Puerta del Vino, où la fin, à partir de la mesure 50, se rapproche d'une organisation mieux hiérarchisée. Cette dissymétrie, totalement absente chez Brahms dont la pièce est au contraire dominée par le stéréotype formel $\mathrm{ABA}$, paraît là encore une caractéristique de la forme debussyste. Il faudrait sans doute pouvoir effectuer de telles expériences sur un grand nombre d'oeuvres du compositeur, avant de pouvoir généraliser à coup sûr, mais que le phénomène apparaisse dans deux pièces aussi différentes que la Cathédrale engloutie et la Puerta del Vino, tend bien à montrer que cette dissymétrie est une dissymétrie du temps musical debussyste qui sous-tend l'organisation thématique elle-même : les " réexpositions " de la phrase en accords d'abord (mesure 72), puis du début (mesure 84) ne sont pratiquement pas perçues à la première audition, et n'apparaissent qu'à un faible niveau dans la hiérarchie à la seconde audition. Nous avons déjà noté ce phénomène pour la Puerta del Vino.

\section{B - Dissymétrie de la macro-structure.}

Les résultats d'ensemble que nous venons d'exposer brièvement, mettent en lumière les principaux aspects de la macrostructure de la Cathédrale.

Tout d'abord, cette macro-structure est faiblement hiérarchisée. Ce caractère apparaît dès la première audition : six points de condensation perceptive (contre deux pour Brahms !) à un seuil $\mathrm{P}=.001$, mais seize points de condensation à un seuil $\mathrm{P}=.01$, ce qui est considérable. De ce point de vue, la Cathédrale apparaît presque plus morcelée que la Puerta (seize points de condensation pour 330 secondes de durée totale de la pièce, alors que la Puerta en présentait sept pour une durée de 175 secondes). Mais elle est cependant probablement plus fortement hiérarchisée que la Puerta, sans s'apparenter pour autant à la structure de l'Intermezzo. En effet, la figure 3 suggère qu'il existe des différences nettes de prégnance perceptives entre les seize points de condensation. En fait, les six points situés à 28", 50"-52", 1'02"-1'03", 1'25"-1'26", 1'49"-1'50" et 3'02"'-3'03" semblent constituer la charpente de la macro-structure, avec d'une certaine façon, un point de condensation perceptive maximum à 1'02"-1'03".

À la première audition, on pourrait donc décrire la pièce de la façon suivante : une introduction assez hétéroclite, le démarrage d'un “ allegro-crescendo " (à 1'02"), cependant rapidement arrêté à 
1'25", puis ensuite, et jusqu'à la fin, une forme évanescente, diffuse, ouverte, dont les contours s'estompent à mesure qu'on s'éloigne du point de condensation maximum, c'est-à-dire du démarrage de l' " allegro-crescendo ». Structure donc totalement dissymétrique, dans laquelle l'amorce de l' " allegro-crescendo » ne tient pas ses promesses ...

Cette façon de présenter et d'analyser la figure 3 met implicitement en jeu, dans le décodage de la macro-structure par les sujets, la référence vraisemblable à un stéréoty pe formel, plus lâche que le schéma classique $A B A$, mais se composant d'une introduction créant une atmosphère, avant le développement de la pièce proprement dite, plus structurée, et où le sujet s'attend à reconnaître des thèmes, une progression jusqu'à un climax central, et peut-être le retour de ces mêmes thèmes en une atmosphère plus calme et plus détendue. On peut penser que le début de la Cathédrale est un peu perçu selon ce schéma par les sujets, mais le beau développement attendu à partir du moment où le rythme s'accélère (mesure 16, 1'02") et modifie l'atmosphère d'attente du début (Debussy ne note-il pas sur la partition " peu à peu sortant de la brume "?), ne vient pas, ou plutôt vient mal, car le climax central, qui pourrait correspondre, du point de vue de l'intensité sonore, à la fameuse phrase des grands accords (entrée à 1'49") n'est plus suivie d'indications perceptives symétriques très prégnantes. Elle-même n'est d'ailleurs pas aussi nettement perçue que l'amorce de l'allegro-crescendo, alors qu'on aurait pu penser que ce grand sommet fût signalé comme un moment-clé par tous les sujets.

Ainsi, à la première audition, la Cathédrale engloutie apparaîtrait comme une forme incomplète, ou plutôt comme une forme fortement dessinée au début, esquissée ensuite. La phénomène est d'autant plus remarquable qu'il était déjà observé à la première audition de la Puerta del Vino, non à la première audition de l'Intermezzo de Brahms. Il s'agirait donc là d'une caractéristique stylistique propre à Debussy. En tout cas, pour la Cathédrale, est-ce là peut-être l'une des raisons pour lesquelles, malgré eux, certains critiques ne parviennent à conduire une analyse sémantique détaillée que sur le début de la partition, même si, dans un commentaire plus général et non linéaire, ils mentionnent ensuite "les orgues lointaines" ou le "choral puissant et grandiose "?

Si cette analyse est exacte, on peut dire alors qu'il y a une sorte d'inadéquation entre le stéréotype formel implicitement utilisé par 
les sujets (et cette hypothèse est fortement suggérée par l'organisation générale de la macro-structure à la première audition), et la structure lue dans la partition de la pièce. Ou plus exactement, le stéréotype ne permet pas aux sujets de saisir en une totalité cohérente, hors de l'écoulement irréversible du temps, l'organisation musicale. Cette distance entre la référence culturelle implicite et l'oeuvre crée alors ce sentiment du temps disloqué qui ne mène nulle part, à la limite immobile parce que le sujet ne peut anticiper ni prévoir, rendu prisonnier de l'instant dont seul, le compositeur, conserve le sens insoupçonné.

Or la seconde audition accentue ce disfonctionnement du stéréotype " introduction - allegro-crescendo », en ce que l'entrée des grands accords est encore moins bien perçue qu'à la première audition, et que c'est un élément thématique secondaire qui détermine le point de condensation perceptive maximum : l'entrée des octaves aigües (mesure 23 à 1'32") à laquelle répond plus faiblement, mais plus nettement que celle des grands accords (à 1'48"), l'entrée des octaves graves (mesure 25 à 1'38"). Il devient d'ailleurs difficile de continuer à parler de point de condensation perceptive maximum : les sept premiers points de condensation peuvent vraiment être considérés comme équivalents (tous significatifs à un seul $\mathrm{P}<.001$ ), et parmi eux apparaissent des ruptures à peine perçues lors de la première audition, ce qui prouve combien la hiérarchie est faible dans tout ce début de la pièce.

Cependant, comme dans la Puerta del Vino, et à l'inverse de ce qui s'était passé dans la pièce de Brahms, il n'y a pas eu de réorganisation sensible de la macro-structure d'une audition à l'autre. Lorsqu'on compare les figures 3 et 4, on s'aperçoit aisément que leur aspect d'ensemble demeure inchangé, et que, d'une certaine façon, tout ce qui a été dit à propos de la première audition se retrouve à la seconde. En particulier, la dissymétrie de la structure est confirmée de manière spectaculaire : non seulement, au début, on observe sept points de condensation pour une durée de 1'30", et seulement quatre points de condensation ensuite, sur une durée de 4', mais en outre, la prégnance des sept premiers points est nettement plus forte que la prégnance de ceux qui suivront. On arrive donc à une structure extrêmement morcelée et peu hiérarchisée au début, moins morcelée ensuite, sans qu'il y ait pour autant une hiérarchie très forte. Si l'on compare les figures 2 et 6 , on se rend compte là encore que les deux pièces de Debussy s'apparentent.

Cependant, on avait noté, à propos de la Puerta del Vino, que 
la seconde audition avait tendance à symétriser le dissymétrique, par l'intervention implicite d'une référence à un stéréotype formel (Imberty 1981) : à la première audition, toute la fin à partir de la mesure 50 ne donnait lieu qu'à très peu de réponses des sujets. A la seconde audition, deux points de condensation perceptive très prégnants apparaissaient à la mesure 79 d'abord, à la mesure 83 ensuite, coïncidant avec la reconnaissance d'un élément entendu au début et alors bien isolé (mesures 21 et 25). Mais en même temps, on notait que cet élément n'est pas un " thème " à proprement parler, et qu'il était perçu essentiellement sur la base de son impact dynamique. Le seul élément véritablement thématique - et un peut traité comme tel - est la mélodie ibérique du début (mesure 5 et suiv.). Or, nous l'avons déjà noté, lorsque celle-ci est " réexposée », elle n'est nullement perçue par les sujets (figure 2, 1'57", mesure 66). Par conséquent, la rééquilibration des points de condensation perceptive au cours de la seconde audition s'est faite uniquement par assimilation à un schème d'ordre, et non par la mise en oeuvre de relations d'ordre formalisées. En l'occurrence, ce schème peut être considéré comme un schème de rémanence, dont le principe est l'assimilation de certains phénomènes sonores passés à des phénomènes présents, sur la base d'une persistance implicite dans la mémoire des images des premiers. Naturellement, cette permanence des images sonores passées dans la mémoire du sujet est renforcée par les auditions successives, et il est normal que l'effet des rémanences ne soit pas ou peu sensible à la première audition. Mais ce surgissement du passé au moment d'une recentration perceptive présente, souligne le caractère ponctuel et parcellaire de ces assimilations : elles n'ont pas pour effet d'unifier et de relier entre elles des zones temporelles plus ou moins larges, mais seulement de faire surgir certains rapprochements dans l'esprit du sujet, rapprochements qui ne concernent que des éléments brefs et isolés. Réminiscences en somme, par réactivation d'un souvenir qui ne concerne pas la trame du flux musical, mais seulement les points saillants qui ont frappé l'auditeur.

Dans le style de Debussy, on peut dire que la macro-structure s'appuie en fait sur ces rémanences, plus fortes et plus prégnantes que les transitions, les retours, les réexpositions. Bien des codas sont construites ainsi, avec l'irruption soudaine d'un thème suspendu sur une harmonie de tonalité différente, souvenir qui remonte d'un lointain passé, et qui ne clôt pas la forme en la ramenant à son point de départ, mais simplement l'arrête dans une 
sorte d'instant éternisé (ex. : La fille aux cheveus de lin, Bruyères), ou avec des lambeaux de thèmes, à la fois imprécis et reconnaissables, qui s'effacent lentement, conduisent l'oeuvre en silence, produisent cette impression de fuite du temps, de dislocation et d'anéantissement que l'on trouve dans Fêtes, Nuages, Canope, dans la Puerta del Vino, à partir de la mesure 79, ou précisément encore dans ce retour à l'octave grave et pianissimo, de la grande phrase en accords de la Cathédrale engloutie, écho lointain de l'espace et du temps.

La symétrisation perceptive de la forme repose donc bien, chez Debussy, sur les réminiscences engendrées par les schèmes de rémanence, et non sur les relations tonales qu'impliquent les stéréotypes classiques auxquels les sujets-auditeurs font probablement référence. L'explication de ce phénomène est sans doute que toute la macro-structure debussyste est essentiellement constituée par les contrastes dynamiques, les oppositions de climat ou d'atmosphère, bref, par l'identification de moments sonores perçus pour eux-mêmes avant d'être insérés dans leur contexte. Les schèmes de rémanence assimilent ces éléments-là, non les éléments thématiques identifiés comme tels, car l'on ne se souvient pas d'un accord ff ou d'un timbre particulier comme on se rappelle un thème au moment de sa réexposition.

Qu'observe-t-on pour la Cathédrale engloutie? Lors de la première audition, sont nettement perçus : l'entrée de l' " allegrocrescendo ", l'entrée des grands accords et le départ, à la mesure 47 , de la reprise du thème exposé à la mesure 7 (nous allons voir s'il s'agit véritablement d'un thème). A la seconde audition, même si l'entrée des grands accords est perçue (à 1'48"), c'est en fait un élément secondaire qui déclenche principalement les réponses des sujets : l'entrée des octaves aigües à 1'32 (mesure 23). Or c'est le battement de la seconde à l'extrême grave (4'14") qui est perçu symétriquement à l'entrée des octaves aigües, et non l'entrée des grands accords "en échò" (4'20"). La symétrie - moins spectaculairement rétablie qu'à la seconde audition de la Puerta se construit à nouveau non sur des thèmes reconnus comme tels, mais bien sur des éléments sonores perçus pour leur qualité propre, leur "couleur", bref, pour leur "timbre». Dans ces conditions, il est probable que la rentrée de l'élément thématique des mesures 7 et suivantes, à l'octave grave, à la mesure $47,-$ qui n'est d'ailleurs pas très prégnante à la seconde audition — n'est pas reconnu comme tel, mais assimilé aux schèmes d'ordre, en fonction du changement de tempo, et du simple fait sans doute, qu'après les 
longues tenues s'évanouissant dans le silence, quelque chose " repart » et relance l'attention.

On retrouve donc à nouveau un point important qui différenciait déjà la macro-structure de la Puerta de celle de l'Intermezzo de Brahms : les sujets ne perçoivent et n'identifient pas des thèmes, mais seulement des éléments dynamiques. Aucun des éléments repérés ne semblent jouer le rôle de thème, puisque, pour la Cathédrale notamment, on ne les retrouve jamais perçus deux fois avec la même intensité tout au long de la pièce. Les " thèmes " sont perçus au début; ensuite, tout paraît toujours imprévisible et nouveau, succession de moments, non écoulement du temps. Et il est facile de se rendre compte que les principaux points de condensation perceptive coïncident avec ces éléments à couleur sonore particulière, qui constituent finalement la base de l'organisation de la macros-structure. Le tableau III présente l'analyse de ces éléments pour les deux auditions, à un seuil de significativité statistique comparable $(.05<\mathrm{P}<.10)$.

Par conséquent, à côté du morcellement du temps musical, de la dissymétrie de la construction formelle (forme " esquissée " ou " estompée en son devenir "), un autre caractère important de l'écriture debussyste se fait jour ici : la recherche de la sonorité, du timbre du piano comme on l'a dit parfois, et cette subtilité chatoyante, dont on aurait pu croire qu'elle était sensible surtout à ceux des auditeurs connaissant bien l'oeuvre de Debussy et capables de la replacer dans l'histoire de la musique, apparaît tellement évident que, même pour des sujets amateurs comme les nôtres, elle constitue la charpente et la raison d'être de l'oeuvre tout entière.

Ces résultats sont suffisamment clairs pour montrer à quel point la démarche que nous avons adoptée peut nous renseigner sur ce qu'est le style d'un compositeur : l'organisation perçue des changements, ruptures, contrastes n'est pas seulement dépendante de mécanismes perceptifs et d'habitudes culturelles dont on a vu l'importance, mais également de la forme musicale elle-même, dans la mesure où celle-ci est construite selon des règles propres que s'est imposé le compositeur et qui viennent s'inscrire à l'intérieur ou, au contraire, à l'encontre des connaissances que le sujet a à priori de l'art musical dans sa culture. Sans doute, pour la Cathédrale, comme pour la Puerta del Vino, les éléments "sonoriels" (Jarocinski 1966) et dynamiques jouent-ils un rôle beaucoup plus important dans la macro-structure que chez Brahms : on pourrait penser qu'il s'agit là d'une « faiblesse » de la 


\begin{tabular}{|c|c|c|c|c|c|c|}
\hline minutage & mesures & $\begin{array}{c}\text { élément } \\
\text { perçu }\end{array}$ & $\begin{array}{l}\text { première } \\
\text { audition }\end{array}$ & $\begin{array}{c}\text { deuxième } \\
\text { audition }\end{array}$ & $\begin{array}{l}\text { aspect sonore } \\
\text { ou dynamique }\end{array}$ & aspect thématique \\
\hline $5 "$ & 2 & $\begin{array}{l}\text { sol grave } \\
3^{e} \text { temps }\end{array}$ & & $\mathbf{x}$ & registre grave après aigü & \\
\hline 7"-8" & 3 & $1^{\text {er }}$ temps & & $\mathbf{x}$ & $\begin{array}{l}\text { modification sonore de } \\
\text { l'accord initial }\end{array}$ & \\
\hline $16 "$ & 5 & $1^{\text {er }}$ temps & & $\mathbf{x}$ & id. & \\
\hline 23" & 6 & $5^{e}$ temps mi & $\mathbf{x}$ & $\mathbf{x}$ & registre aigu et tenue longue & $\begin{array}{l}\text { fin de la série } \\
\text { d'accords. (A) }\end{array}$ \\
\hline 26" & 7 & $1^{\text {er }}$ temps & $\mathbf{x}$ & $\mathbf{x}$ & $\begin{array}{l}\text { lâcher de la pédale } \\
\text { (arrêt de la résonance) }\end{array}$ & \\
\hline $27 "-28 "$ & 7 & $\begin{array}{l}3^{e} \text { ou } \\
5^{e} \text { temps }\end{array}$ & $\mathbf{x}$ & $\mathbf{x}$ & $\begin{array}{l}\text { nouvelle phrase souvent } \\
\text { perçue sur le do dièze }\end{array}$ & $\begin{array}{l}\text { nouvel élément } \\
\text { thématique. (B) }\end{array}$ \\
\hline 38" & 10 & $3^{e}$ temps & & $x$ & $\begin{array}{l}\text { tension harmonique due au } \\
\text { la dièze }\end{array}$ & \\
\hline $50 "$ & 13 & $3^{\mathrm{e}}$ temps & $\mathbf{x}$ & $\mathbf{x}$ & retour du registre grave & \\
\hline $55 ”$ & 15 & $1^{\text {er }}$ temps & $\mathbf{x}$ & $\mathbf{x}$ & $\begin{array}{l}\text { sommet aigu de la courbe } \\
\text { mélodique des quintes et/ou } \\
\text { accord grave }\end{array}$ & \\
\hline 1'02"-1'03" & 16 & $1^{\text {er }}$ temps & $\mathbf{x}$ & $\mathbf{x}$ & $\begin{array}{l}\text { mouvement plus rapide - } \\
\text { triolets de la main gauche }\end{array}$ & $\begin{array}{l}\text { nouvel élément théma- } \\
\text { tique, nouvelle partie (C) }\end{array}$ \\
\hline 1'10" & 10 & $4^{e}$ temps & $\mathbf{x}$ & $\mathbf{x}$ & (crescendo de l'interprète (?) & \\
\hline 1'13"-1'14" & 19 & $2^{e} / 3^{e}$ temps & $\mathbf{x}$ & $\mathbf{x}$ & $\begin{array}{l}\text { modulation en mi bémol } \\
\text { (changement de couleur) }\end{array}$ & \\
\hline
\end{tabular}




\begin{tabular}{|c|c|c|c|c|c|c|}
\hline minutage & mesures & $\begin{array}{l}\text { élément } \\
\text { perçu }\end{array}$ & $\begin{array}{l}\text { première } \\
\text { audition }\end{array}$ & $\begin{array}{l}\text { deuxième } \\
\text { audition }\end{array}$ & $\begin{array}{l}\text { aspect sonore } \\
\text { ou dynamique }\end{array}$ & aspect thématique \\
\hline & 18 & $4^{e}$ temps & & & $\begin{array}{l}\text { dissonance de } 7^{e} \text { majeure } \\
\text { à la main gauche }\end{array}$ & \\
\hline 1'20" & 21 & $1^{\mathrm{er}}$ temps & & $x$ & groupe doubles croches m.g. & $\begin{array}{l}\text { groupe à valeur } \\
\text { thématique (?) (C') } \\
\text { depuis mesure } 19, \text { m.g. }\end{array}$ \\
\hline 1'22" & 21 & $4^{\mathrm{e}}$ temps & & $x$ & id. & \\
\hline $1 ' 25 "$ & 22 & $1^{\mathrm{er}}$ temps & $\mathbf{x}$ & $\mathbf{x}$ & retour aux valeurs longues & $\begin{array}{l}\text { thème précédent en } \\
\text { valeurs longues }(C)\end{array}$ \\
\hline $\begin{array}{l}\text { 1'26" } \\
\text { 1'27" }\end{array}$ & 22 & $\begin{array}{l}2^{\mathrm{e}} \text { temps } \\
3^{\mathrm{e}_{\text {temps }}}\end{array}$ & $\mathbf{x}$ & $\mathbf{x}$ & chaque accord & \\
\hline 1'32" & 23 & $3^{e_{-4}} e$ temps & $\mathbf{x}$ & $\mathbf{x}$ & octaves aigües & $\begin{array}{l}\text { groupe à valeur } \\
\text { thématique (?) (C”) }\end{array}$ \\
\hline 1'38"-1'39"' & 25 & $3^{e_{-4}} e_{\text {temps }}$ & & $x$ & octaves graves & le même groupe (C") \\
\hline 1'48"'-1'49"' & 28 & $1^{\text {er }}$ temps & $x$ & $x$ & ut grave & \\
\hline 1'50" & 28 & $3^{\mathrm{e}}$ temps & $\mathbf{x}$ & $\mathrm{x}$ & & $\begin{array}{l}\text { entrée des grands } \\
\text { accords (D) }\end{array}$ \\
\hline 2'05" & 32 & $3^{\mathbf{e}}$ temps & $x$ & $x$ & ut grave & \\
\hline 2'24"-2'25" & 37 & $3^{\mathrm{e}}$ temps & $\mathbf{x}$ & $x$ & ut grave & \\
\hline 2’33" & 39 & $3^{\mathrm{e}}$ temps & $\mathbf{x}$ & $x$ & decrescendo & $\begin{array}{l}\text { annonce la fin de la } \\
\text { phrase des grands } \\
\text { accords (D) }\end{array}$ \\
\hline 2'38"-2'39"' & 40 & $3^{e}$ temps & $\mathbf{x}$ & $x$ & ut grave & élément thématique $(\mathrm{C})$ \\
\hline
\end{tabular}




\begin{tabular}{|c|c|c|c|c|c|c|}
\hline minutage & mesures & $\begin{array}{l}\text { élément } \\
\text { perçu }\end{array}$ & $\begin{array}{l}\text { première } \\
\text { audition }\end{array}$ & $\begin{array}{c}\text { deuxième } \\
\text { audition }\end{array}$ & $\begin{array}{l}\text { aspect sonore } \\
\text { ou dynamique }\end{array}$ & aspect thématique \\
\hline $\begin{array}{l}23^{\prime \prime}-44^{\prime \prime} \\
-45^{\prime \prime}-46 "\end{array}$ & $41-42$ & $\begin{array}{l}\text { tous les } \\
\text { temps } \\
\text { frappés }\end{array}$ & & $x$ & $\begin{array}{l}\text { principalement à } 2 \text { ' } 46^{\prime \prime} \text { le si } \\
\text { bémol grave et la seconde à } \\
\left.\text { l'extrême aigu ( } 3^{e_{t}} \text { mes. } 41\right)\end{array}$ & \\
\hline 3'02"-3'03" & 46 & $3^{e}$ temps & $\mathbf{x}$ & $x$ & sol dièze grave longue tenue & fin d'une période \\
\hline 3'18" & 51 & $3^{e}$ temps & $x$ & $x$ & $\begin{array}{l}\text { modulation et harmonisa- } \\
\text { tion du thème précédent }\end{array}$ & $\begin{array}{l}\text { entrée du thème }(B) \text { mais } \\
\text { l'entrée précédente } \\
\text { n'était pas perçue } \\
\text { (mes. 47) }\end{array}$ \\
\hline 3'25" & 54 & $1^{\mathrm{er}}$ temps & $x$ & & dissonance $2^{e} \min$. & \\
\hline 3'32" & 56 & $3^{e}$ temps & & $x$ & crescendo & \\
\hline 3'37"'-38" & 58 & $5^{\mathrm{e}}$ temps & & $x$ & id. & \\
\hline $3{ }^{\prime} 30^{\prime \prime}-40 "$ & 59 & $1^{\mathrm{er}}$ temps & $x$ & & id. et changement de registre & \\
\hline $3^{\prime} 44^{\prime \prime}-45^{\prime \prime}$ & 61 & ${ }_{1}^{\text {er }}$ temps & & $x$ & sommet du crescendo & \\
\hline $3 ' 50 "$ & $\begin{array}{c}63 \\
(62\end{array}$ & $\begin{array}{l}1^{\mathrm{er}} \text { temps } \\
5^{\mathrm{e}} \text { temps) }\end{array}$ & & $x$ & $\begin{array}{l}\text { decrescendo perçu en retard } \\
\text { (à cause de sa brusquerie?) }\end{array}$ & \\
\hline 3'55" & 64 & $5^{e}$ temps & $\mathrm{x}$ & $x$ & & $\begin{array}{l}\text { élément répétitif à valeur } \\
\text { thématique secondaire }\end{array}$ \\
\hline 3’58" & 65 & $5^{e}$ temps & & $x$ & & id. \\
\hline 4'05"-06" & 68 & ${ }_{1}^{\mathrm{er}}-\mathrm{e}^{\mathrm{e}}$ temps & $\mathbf{x}$ & $x$ & seconde et triton graves & \\
\hline 4'14"-15" & 70 & $3^{e}$ temps & & $x$ & trémolo grave & \\
\hline 4'20"' & 72 & $3^{\mathrm{e}}$ temps & & $x$ & & $\begin{array}{l}\text { grands accords } \\
\text { "en écho" (D) }\end{array}$ \\
\hline
\end{tabular}




\begin{tabular}{|c|c|c|c|c|c|c|}
\hline minutage & mesures & $\begin{array}{c}\text { élément } \\
\text { perçu }\end{array}$ & $\begin{array}{c}\text { première } \\
\text { audition }\end{array}$ & $\begin{array}{c}\text { deuxième } \\
\text { audition }\end{array}$ & $\begin{array}{l}\text { aspect sonore } \\
\text { ou dynamique }\end{array}$ & aspect thématique \\
\hline 5’05"-06" & 84 & $2^{\mathbf{e}}$ temps & $\mathbf{x}$ & $\mathbf{x}$ & changement de registre & $\begin{array}{l}\text { accords de quinte du } \\
\text { début (A) }\end{array}$ \\
\hline 5'07" & 85 & $1^{\text {er }}$ temps & & $\mathbf{x}$ & & $\begin{array}{l}\text { terminaison de la courbe } \\
\text { mélodique des accords } \\
\text { de quinte. }\end{array}$ \\
\hline $5 ’ 15^{\prime \prime}$ & 86 & $5^{\mathrm{e}}$ temps & & $\mathbf{x}$ & accord de quinte sur ut grave & \\
\hline
\end{tabular}

\section{Tableau III}

Analyse des principaux points de condensation perceptive, $1^{\mathrm{e}}$ audition et $2^{e}$ audition. de la Cathédrale engloutie (seuil $P$ choisi sur les figures 3 et $4: P=.07$ ) 
perception des sujets-auditeurs. Mais, précisément dans la mesure où ce phénomène se retrouve à l'audition de plusieurs pièces de Debussy, peut-on aussi penser que cette «faiblesse " est provoquée par la forme elle-même, telle que l'a conçue le compositeur. Déjà, à propos de la Puerta, nous avons montré que la structure de la pièce suppose en fait un décalage constant entre la thématique et la dynamique, de telle sorte que celle-ci passe au premier plan de la forme. Chez Brahms, la construction thématique élaborée et travaillée en de longs développements de petites unités variées à l'infini, coïncide avec le mouvement dynamique d'ensemble et l'ordre de succession de ces développements, unifiant ainsi le flux temporel. Chez Debussy au contraire, il y a décalage constant entre l'évolution des thèmes et l'évolution dynamique, et c'est par cette originalité que Debussy a pu se dégager des canons formels stéréotypes : encore une fois, sa musique est une musique de l'instant et non du devenir, et la faible hiérarchisation de la macrostructure ainsi que la prédominance structurale des contrastes et des ruptures en sont les manifestations les plus claires.

\section{La composante sémantique de la macro-structure de la Cathédrale}

\section{A - Définition}

L'étude de la macro-structure est donc susceptible de nous éclairer à la fois sur les processus perceptifs de décodage du style, et sur la fonction du temps musical comme représentation symbolique des vécus du temps en général.

En effet, les hiérarchies des changements, leur densité, leur prégnance, leurs qualités constituent des architectures du temps dont nous venons de voir combien elles diffèrent profondément d'une époque à l'autre, d'un compositeur à l'autre. C'est pourquoi il nous semble qu'il convient d'aborder la sémantique d'une pièce musicale dans cette perspective : son organisation temporelle porte sens par rapport aux vécus individuels ou collectifs du temps. L'expérience auditive des changements, des ruptures et des contrastes renvoie à l'expérience du temps, et nous pouvons formuler l'hypothèse que tout discours sur l'oeuvre, toute association verbalisée plus ou moins spontanée, plus généralement, toute interprétation - y compris l'interprétation-exécution - témoigne de cette représentation des vécus inconscients du temps par le temps musical.

Toujours à propos de l'Intermezzo de Brahms et de la Puerta 
del Vino, nous avons montré (Imberty 1981) que si l'on demande à des sujets musiciens de décrire les séquences principales des deux pièces obtenues par le découpage auditif expérimental, les réponses sémantiques (à contenu extra-musical) prédominent largement par rapport aux réponses techniques ou formelles, bien que la consigne ne demandait nullement des sujets de telles réponses. Plus exactement, si l'on examine le contenu de l'ensemble des réponses fournies dans ces conditions, on se rend compte qu'à côté de réponses purement techniques, les augmentations ou diminutions de caractères globaux, les reprises, les répétitions ou retours, les changements de climat, les contrastes de volume sonore donnent lieu à des réponses qui supposent un contenu extra-musical, au moins potentiel : des termes comme "calme " ou "tendre " font bien partie de ces adjectifs qui constituent des connotations de la forme musicale autant que des significations particulières associées. Mais on reconnaît là comment certaines qualités de la forme sonore sont un encodage, dans un système musical déterminé, d'expériences temporelles plus larges auxquelles elles sont assimilées, et par lesquelles elles prennent des significations extra-musicales. Toutes les réponses désignant des qualités de cet ordre - précisément véhiculées par les éléments dynamiques - ont trait aux vécus de la durée, définie comme plus ou moins forte densité d'évènements sonores, accélération ou ralentissement qui ne sont pas seulement ni essentiellement liés au tempo, mais à tout ce qui donne l'illusion d'une augmentation de la masse sonore, de sa complexité, ou, inversement, d'une diminution et d'une simplification.

Un exemple particulièrement net était celui de ce qu'on peut appeler la coda de la Puerta del Vino : les réponses indiquaient surtout une sorte de "sérénité " ou d' "immobilisation " et de " calme ". Le passage à l'octave aigüe (mesure 78) est en effet caractérisé par ce ralentissement du temps (sans ralentissement objectif du tempo), par cette "immobilisation " ou cette "sérénité » qui détend la densité de la durée et de l'écriture. Le passage à l'octave aigüe n'est pas apparu aux sujets seulement comme un changement de registre, mais il a pris pour eux la signification d'une sorte d'ouverture et d'immobilisation du temps musical que le dernier arpège vient briser brusquement. Ce changement perçu est donc un vecteur dynamique qui oriente le fonctionnement des schèmes d'ordre à travers la réorganisation perceptive qu'il suscite, et qui détermine un comportement sémantique donné en réponse à toute la séquence qu'il introduit. Ce comportement, 
explicite ou implicite selon la consigne expérimentale, traduit l'effet émotionnel provoqué par l'assimilation du changement de registre et du contexte dans lequel il intervient aux schèmes de l'activité représentative symbolique, en même temps qu'aux schèmes de la perception du temps (ordre et relation d'ordre).

Il est donc possible de concevoir l'existence psychologique et non sémiologique ${ }^{2}$ - d'une composante sémantique de la macrostructure, composante qui peut être définie comme ensemble des réponses sémantiques potentielles dont le contenu est prédéterminé par les assimilations des vecteurs dynamiques aux schèmes de la perception du temps. Cette composante sémantique relève non du code musical lui-même (même si certaines significations sont conventionnelles et historiquement datées), mais de la perception d'éléments non codés, plus diffus, et d'une assimilation de ces éléments à des significations temporelles. Il est même sans doute difficile de faire un inventaire définitif de ces éléments, car leur appartenance à la composante sémantique dépend de la perception de leur contexte et de leurs relations avec l'ensemble. Là où ils sont repérés par les sujets, c'est qu'ils sont perçus comme essentiels au déroulement temporel de l'oeuvre ; là où ils n'ont pas été identifiés par les sujets, alors même que l'examen de la partition les met en évidence, c'est qu'ils n'y jouent pas ce rôle pour eux. Le sens naît de la dynamique perceptive et de l'intention créatrice elle-même sous-jacente à l'organisation temporelle. Nous retrouvons ici - mais de manière très différente - cette totalité dynamique, cette forme concrète dont nous a entretenu si longuement Deliège (1984). Mais elle est d'abord de nature expressive et sémantique, pas seulement perceptive.

B - L'expérience d'association verbale à la Cathédrale engloutie.

Nous en venons donc à l'expérience qui devait constituer le point de départ de cette étude : les significations que maints critiques musicaux croient reconnaître dans le prélude de Debussy, à partir de ce que suggère son titre, sont-elles reconnues spontanément par les auditeurs, indépendamment de ce titre ? Mais on voit que la perspective est en réalité différente : les significations attendues dans l'expérience doivent, en fonction de tout ce qui précède, d'abord concerner la structure temporelle de la pièce et ce que cette structuration évoque pour les sujets, ce à quoi elle renvoie du point de vue des expériences émotionnelles inconscientes du temps, à travers l'organisation stylistique telle qu'elle vient d'être décrite. 
Le déroulement de l'expérience était le suivant : après les deux auditions expérimentales de la pièce, les sujets l' écoutaient une nouvelle fois, et avaient pour tâche d'associer librement des significations aux principales séquences qu'ils avaient eux-mêmes découvertes lors de la première audition expérimentale. L'expérimentateur leur indiquait donc les divisions au cours de cette nouvelle audition, d'après les réponses fournies lors de la première.

\section{C - Les résultats.}

1) Le dépouillement a été réalisé par deux juges indépendants qui ont procédé à une classification des réponses par catégories sémantiques. Les classifications ont été ensuite confrontées et se sont avérées très concordantes dans l'ensemble : les figures 6 à 8 donnent le nombre de réponses par catégorie et par séquence musicale, le découpage de la pièce correspondant à celui donné à la figure 3 , au seuil de $P=.01$ (valeur critique 6), corrigé par les points de condensation perceptive les plus saillants à la seconde audition (figure 4, 08", 16", 1'32", et 5'15"). Le choix de ce découpage résulte du fait de la consigne donnée aux sujets : ils associaient leurs réponses verbales aux séquences principales fournies lors de la première audition. Toutefois, en ce qui concerne le début de la pièce (séquence à 08 " et 16 "), quelques sujets ont indiqué des réponses différenciées entre 00 " et 28 ", sous forme successive ("d'abord...", " puis..."). Il en a été tenu compte dans le dépouillement.

Il est enfin intéressant de noter que les sujets qui ont, dès la première audition expérimentale, perçu un grand nombre de changements et de ruptures, n'ont jamais établi de parallèle rigoureux entre ce découpage et leurs réponses sémantiques : la plupart du temps, leurs réponses sémantiques définissent des séquences plus larges qui recoupent les séquences présentées dans les figures 6 à 8 . Il est donc clair que toute réponse perceptive (changement perçu) ne donne pas lieu à une induction de significations. À cet égard, le découpage de la pièce, lors de la seconde audition, n'est sémantiquement pertinent que dans la mesure où il ne représente pas une réorganisation perceptive importante par rapport à la première audition. Plus exactement, on observe sur la figure 3 (première audition), certaines indications de changement non statistiquement significatives, reprises par les mêmes sujets à la seconde audition (où elles sont cette fois significative), sans que pour autant elles reçoivent une interpréta- 
tion sémantique de la part de ces sujets : c'est le cas pour la rupture perçue à 2'05" par exemple, de même pour la rupture perçue à 2'33" ou pour celle située à 3 ' 55 ".

Il s'agit presque toujours d'éléments répétés ou répétitifs qui conservent une valeur structurale tout au long de leurs répétitions, mais dont l'interprétation sémantique reste globale, quelle que soit cette structure. Il en est ainsi de l'ut grave qui marque, à 1'49", l'entrée des grands accords parfaits parallèles, la progression vers le sommet d'intensité de la phrase (ut grave répété à 2'05"), puis l'extinction après la chute sur la tonique, à 2 ' 38 ". Du point de vue structural, les fixations perceptives correspondent nettement à la répétion de cette note-pédale, mais sémantiquement, seule la première et la dernière frappe délimitent la période. Quant à la rupture perçue à 2'33", elle correspond à l'amorce du decrescendo, qui, lui non plus, n'a pas de valeur sémantique pour les sujets, bien qu'il soit nettement perçu. De même, à 3'55", l'amorce des accords de $7^{\circ}$ de dominante enchaînés parallèlement n'a, elle non plus, aucune valeur de signification, bien que, structuralement, cet épisode soit nettement isolé au moins à la second audition.

2) Les réponses formelles, d'ordre purement technique ou appréciatif, sont assez peu nombreuses et sont principalement le fait de deux sujets qui, à eux seuls, en fournissent 19 sur un total de 24. L'un d'eux est instrumentiste amateur de bon niveau, l'autre n'a aucune culture musicale (il décrit ce qu'il entend par des réponses $\mathrm{du}$ type "plus fort, plus lent, note grave, etc ... »). Nous retrouvons là un fait constaté par Francès (1958), puis par nousmême (Imberty 1979) : l'attitude sémantique constitue bien l'une des attitudes d'écoute les plus répandues, et l'explicitation de cette attitude dans des réponses sémantiques associées est aisée chez la plupart des sujets.

3) Les réponses de mouvement (description de mouvements ou d'absence de mouvement) sont largement utilisées et concernent la plupart des aspects dynamiques de la forme sonore : accélération ou diminution du tempo et de la vitesse, oppositions de figures rythmiques, progressions d'intensité sonore. Ces réponses résultent de l'assimilation de cette dynamique aux schèmes temporels d'ordre. Le rôle de ces éléments liés au tempo, aux rythmes, aux progressions d'ensemble, qu'elles soient mélodiques ou harmoniques, est d'enclencher des interprétations significantes, la verbalisation d'une représentation de mouvement facilitant souvent les expressions de significations plus complexes (réponses iconiques ou aquatiques) Ces réponses témoignent donc 

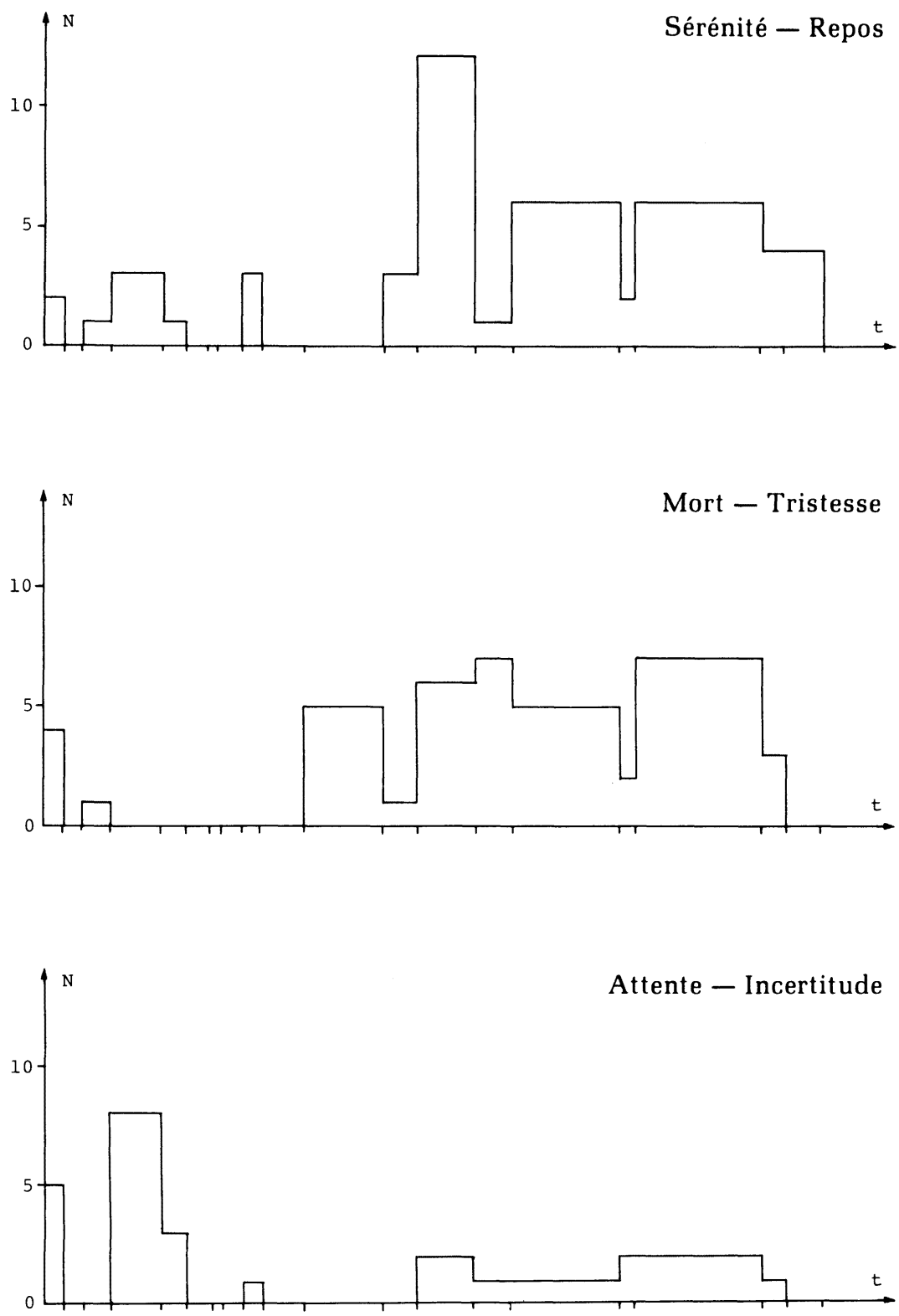

Figure 6

Réponses sémantiques associées au cours de l'audition de la Cathédrale engloutie (en abscisse, les séquences et leur durée. en ordonnée le nombre de réponses pour chaque séquence). Classement par catégories de réponses. 

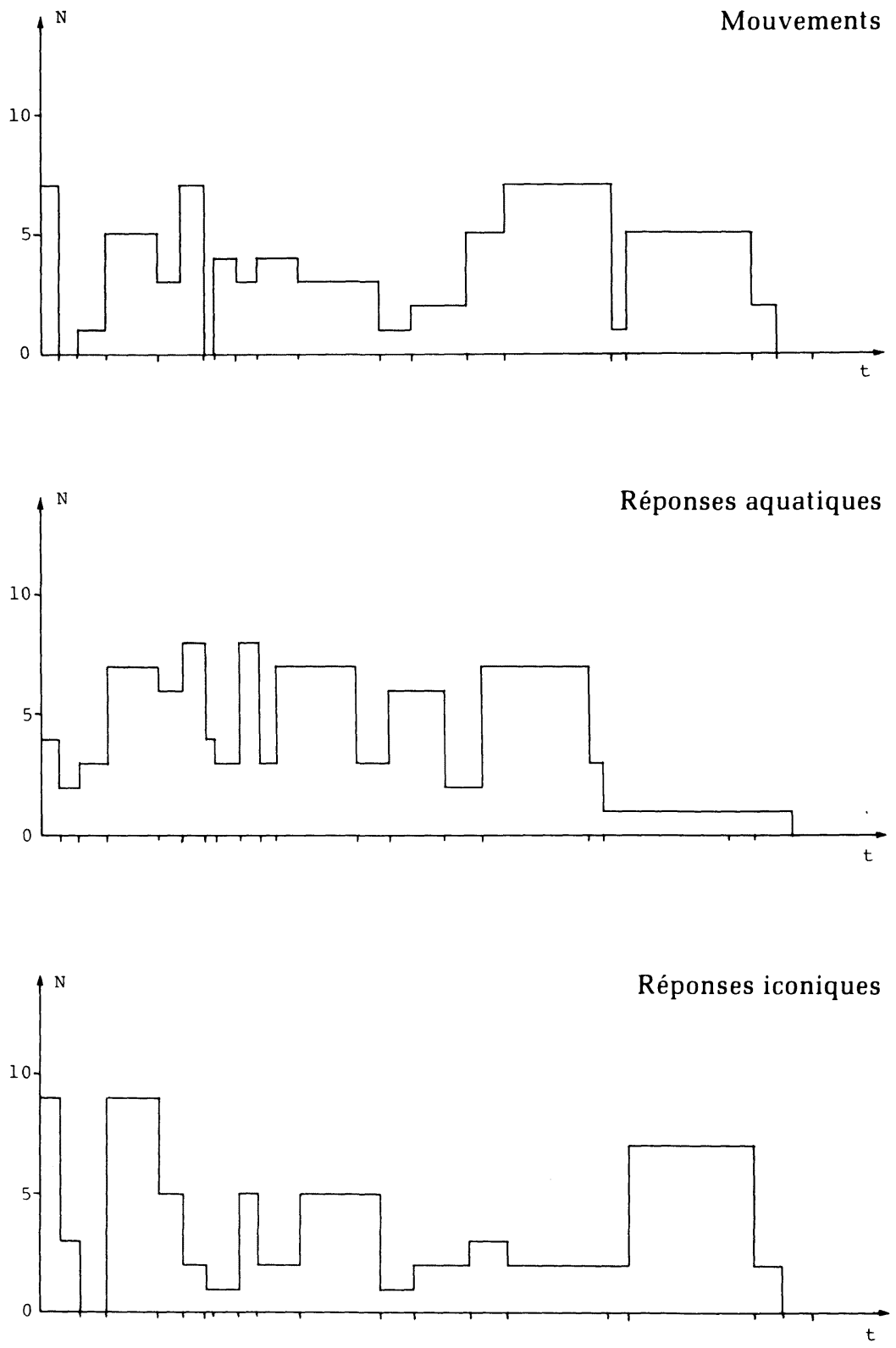

Figure 7

Réponses sémantiques associées au cours de l'audition de la Cathédrale engloutie (en abscisse, les séquences et leur durée, en ordonnée le nombre de réponses pour chaque séquence). Classement par catégories de réponses. 

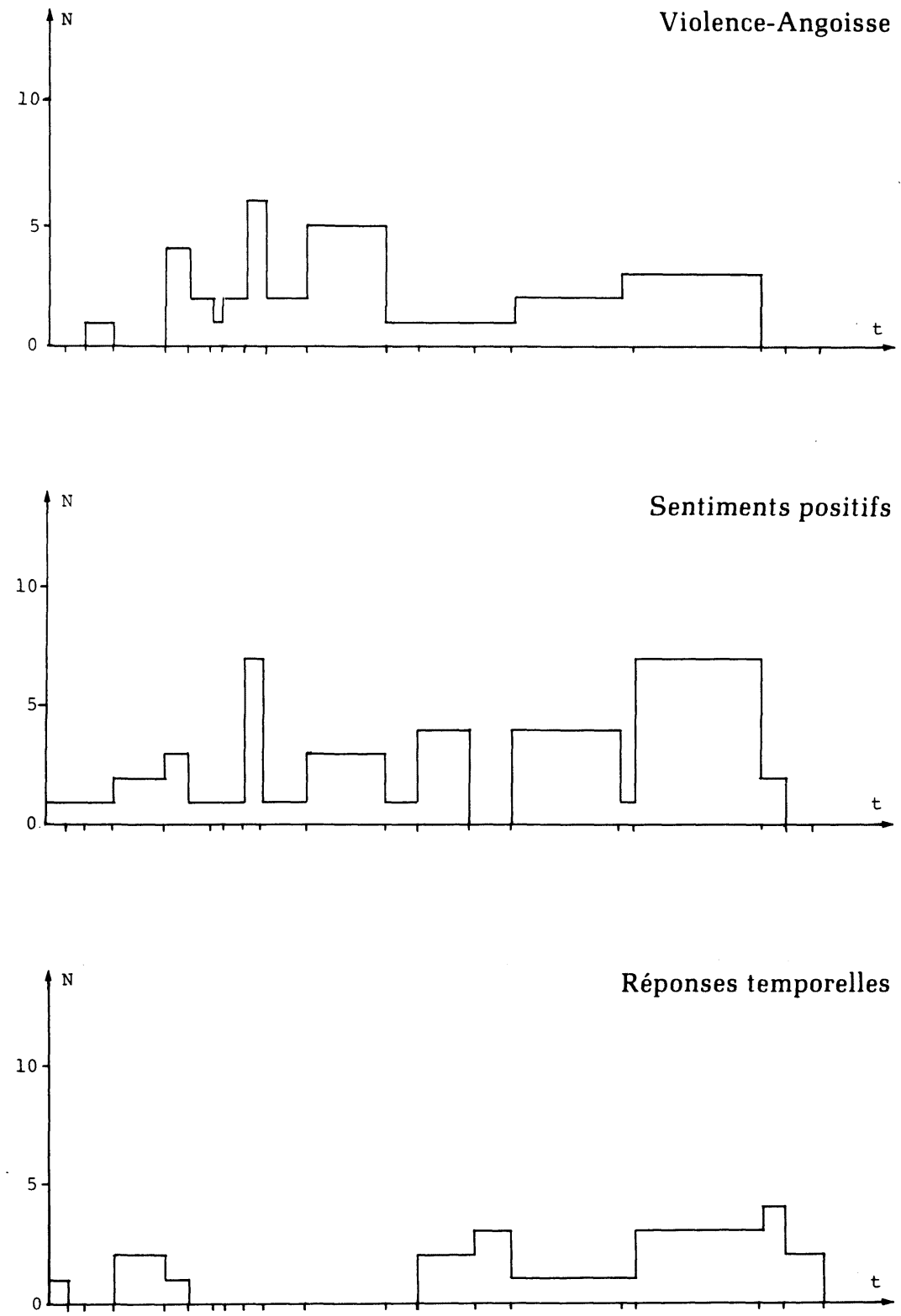

Figure 8

Réponses sémantiques associées au cours de l'audition de la Cathédrale engloutie (en abscisse, les séquences et leur durée, en ordonnée le nombre de réponses pour chaque séquence).

Classement par catégories de réponses. 
largement de ce que nous avons appelé ci-dessus la composante sémantique de la macro-structure, les vecteurs dynamiques de la forme créant le lien nécessaire entre les schèmes d'ordre et les représentations multiformes. La courbe des réponses de mouvements (figure 7) se lit fort bien de ce point de vue : toute la première partie où dominent les mouvements proprement dits (c'est le démarrage de l' "allegro ", pour reprendre notre analyse précédente), puis le ralentissement des mouvements, leur effacement, leur absence, marquent le statisme des grands accords ; à partir de 3'02" et jusqu'à 4'02" un nouvel élan entraîne de nouveau un grand nombre de réponses de mouvements, et à la fin, ces mêmes mouvements sont notés par les sujets comme "s'effaçant", "disparaissant ", s'arrêtant. On retrouve là l'idée selon laquelle ces réponses ne sont pas liées à tel ou tel élément musical précis, mais dépendent à la fois des rythmes, du tempo et de ses fluctuations, de l'intensité sonore, et même des aspects les plus globaux de l'harmonie.

4) Les réponses iconiques, relativement nombreuses, ont des contenus complexes et variés : elles constituent des métaphores de la forme musicale influencées par des habitudes culturelles et des associations-clichés qu'il est difficile d'interpréter plus avant. On peut seulement remarquer que ces réponses, plus nombreuses que pour la Puerta del Vino, décrivent surtout des atmosphères qui correspondent par ailleurs à une expressivité émotionnelle particulière : avec les réponses de sérénité et de repos par exemple, on voit revenir des images telles que "vol d'hirondelles", ou " le soleil se lève ", ou " jardin oriental ". Cependant, d'autres sont plus complexes et probablement trop individuelles pour être rapportées à des aspects précis de la forme musicale ( "artiste au piano », " jeux d'enfants ", " Chine », " film : scène de nuit " ). Il nous paraît en tout cas que toute interprétation de ces réponses est risquée, voire totalement arbitraire si l'on s'en tient à leur littéralité.

5) Les réponses aquatiques, également nombreuses, constituent une catégorie importante. Apparemment, il serait aisé de leur réserver, dans l'interprétation des résultats de l'expérience, le même sort qu'aux réponses iconiques. Cependant, deux faits sont à noter : tout d'abord leur unité thématique marquée (le thème de la mer y est prédominant), et leur répartition tout au long de la pièce ; ensuite, si on compare les résultats obtenus ici et ceux obtenus pour la Puerta del Vino et l'Intermezzo de Brahms, on remarque que : 
- la Cathédrale engloutie a donné lieu à ces réponses aquatiques en assez grand nombre, alors qu'on ne peut les imputer à de nombreux arpèges et traits balayant toute l'étendue du clavier (ces arpèges et ces traits motivaient les quelques réponses de cet ordre données à propos de l'Intermezzo de Brahms).

- la Cathédrale engloutie a donné lieu à ces mêmes réponses alors qu'elles sont totalement absentes des associations sémantiques à la Puerta del Vino.

Il y a donc là un fait : la Cathédrale évoque chez les sujets les images de l'eau, bien que ceux-ci ne connaissaient ni l'oeuvre, ni son titre. Nous avions déjà remarqué, dans notre étude sur les Préludes pour le piano (Imberty 1975, 1976, 1981) que le thème fondamentale de la poétique de Debussy est bien celui de l'eau. Nous proposerons à nouveau une analyse de ce phénomène plus loin. Remarquons seulement que la présence massive de ces réponses aquatiques, alors même qu'on ne les trouvait pas dans la Puerta, suffit à montrer que les titres de Debussy, dans ces fameux Préludes, n'ont rien d'arbitraire, contrairement à ce qui a parfois été affirmé. Mais sans doute a-t-on confondu une fois encore entre la littéralité d'un titre ou d'une image, et son pouvoir d'évocation, beaucoup plus diffus et multiforme : si, en plaçant ses titres à la fin de chaque pièce, Debussy n'a pas voulu influencer l'auditeur, cela ne signifie pas que ses choix étaient arbitraires, ni que ce que la pièce évoque spontanément chez l'auditeur ne puisse avoir aucun rapport avec ce titre. En bref, la Cathédrale ne raconte peut-être pas la légende de la ville d'Ys, sauf pour ceux qui connaissent à la fois l'oeuvre musicale et la légende, mais elle évoque de confuses et riches images d'eau que le titre peut aider à cristalliser autour de certains épisodes de la légende.

Dans l'immédiat, notons, pour ce qui va suivre, que ces réponses peuvent être assez facilement réparties en réponses à " tonalité " positive (avec connotations de sérénité et de repos), et en réponses à " tonalité " négative (avec connotations de violence et d'angoisse). Ceci montre combien toutes les nuances expressives de la pièce sont aisément traduites par les sujets à travers ce thème de l'eau et de la mer.

6) Les réponses émotionnelles se regroupent en plusieurs catégories : sérénité-repos ; sentiments positifs ; mort-tristessenostalgie ; violence-angoisse ; attente-incertitude ; réponses temporelles (marquant l'écoulement du temps sans attente ni incertitude). Le contenu de chaque catégorie est relativement homogène et défini. 
Ce sont principalement les réponses de sérénité et repos, de mort et de tristesse qui caractérisent la pièce. Ceci est conforme à son tempo plutôt lent, mais ce qui frappe, à l'examen de la répartition de ces deux catégories de réponses, c'est qu'en définitive le climat d'ensemble paraît assez ambivalent (figure 9). Or nous avons montré ailleurs (Imberty 1981) que l'ambivalence émotionnelle n'est pas la caractéristique essentielle de l'univers debussyste. Ou bien la Cathédrale fait exception, et par cette ambivalence, se rattache davantage au romantisme (poèmes pour piano avec programme obligé), ce qui expliquerait alors sa relative " popularité ». Ou bien l'analyse que l'on peut faire à partir de ces seules réponses est incomplète.

C'est pourquoi nous avons tenté de dresser un profil « moyen » du climat émotionnel de la pièce, en regroupant d'une part, toutes les réponses à contenu positif, d'autre part, les réponses à contenu négatif ou destructeur. Cette procédure nous avait permis de dresser un profil analogue pour la Puerta del Vino, reproduit à la figure 10 . Nous avons donc regroupé d'une part les catégories " sérénité-repos ; sentiments positifs ; réponses temporelles ; réponses aquatiques ayant trait aux images de l'eau claire et calme ", d'autre part les catégories " mort-tristesse-nostalgie ; violence-angoisse; attente-incertitude; réponses aquatiques ayant trait aux images de la tempête et de l'eau démontée ". La figure 9 présente ainsi le profil de la Cathédrale engloutie.

Ce profil apparaît globalement plus symétrique que celui de la Puerta, mais les contrastes sont tout aussi marqués entre les périodes sombres et angoissantes et les périodes sereines et lumineuses. Mais on remarque aussi que la progression d'ensemble est ascendante, comme dans la Puerta, allant d'un début sombre et triste, parfois violent (jusqu'à la fin de la phrase des grands accords parfaits parallèles), vers une fin apaisée, sereine et immobile dans une sorte de complète intemporalité. L'apparence de symétrie s'estompe donc dans cette dissymétrie ascensionnelle, propre à l'univers debussyste, et qui témoigne d'un déni du temps et de la mort, sous forme d'un refus des codas tragiques du romantisme. Ce déni de la fuite irréversible du temps apparaît notamment dans l'impression produite sur les sujets par les toutes dernières mesures et leurs sonorités lumineuses d'ut majeur. 


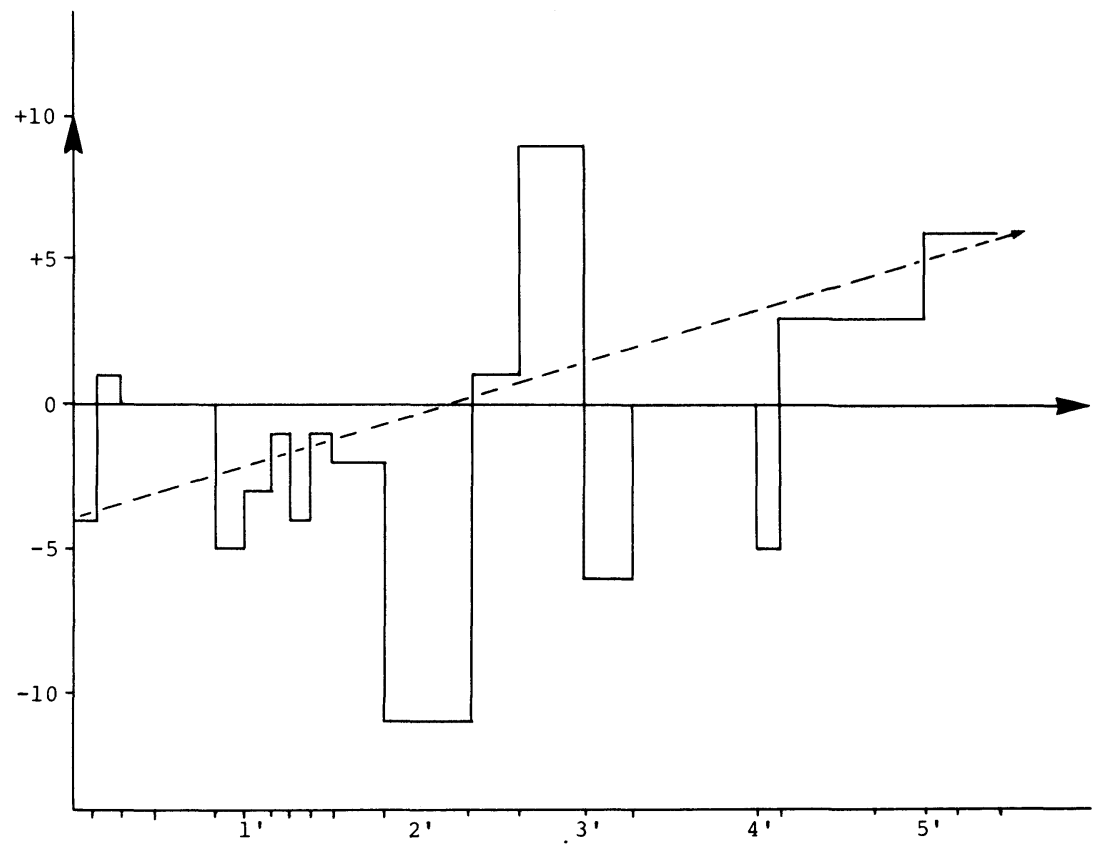

Figure 9

Profil émotionnel " moyen " de la Cathédrale engloutie

+ Sérénité ; repos ; sentiments positifs ; réponses temporelles ; réponses aquatiques, "eaux claires"

- Mort-tristesse ; violence-angoisse ; attente-incertitude ; réponses aquatiques, " tempêtes" 


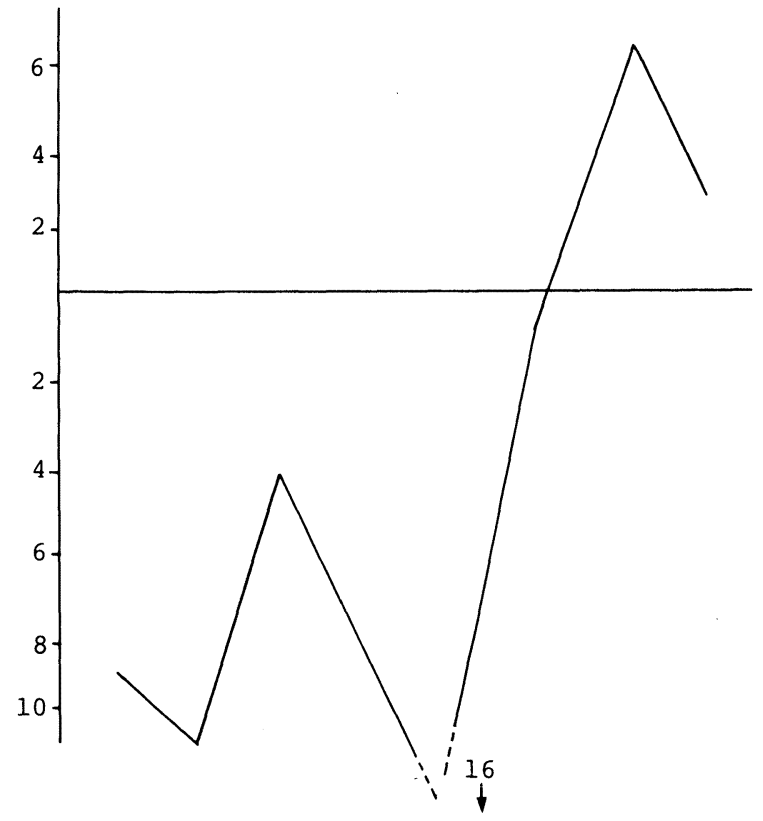

Figure 10a

Profil « moyen » du climat émotionnel de la Puerta del Vino

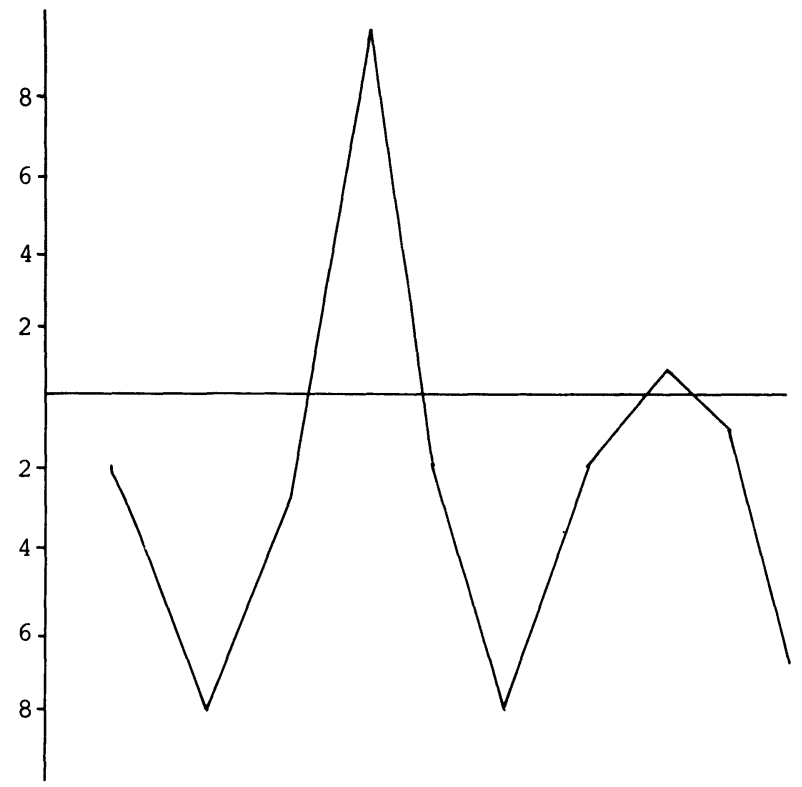

Figure 10b

Profil " moyen " du climat émotionnel de l'Intermezzo de Brahms 
D - De la forme au contenu : le poétique de l'eau dans la Cathédrale.

1) La dernière remarque est intéressante : en effet, les dernières mesures, à partir de 5'05" (mesure 84), reprennent les fameux accords de quintes parallèles du début, et pourtant, du point de vue sémantique, leur signification apparaît totalement différente. On retrouve là le lien profond entre le sens et la structure, plus exactement entre le sens et la forme que prend, dans le style, l'expérience intérieure du temps. Nous avons largement développé ailleurs (Imberty 1981) que la sémantique musicale est une sémantique du temps, et que la forme musicale est une représentation symbolique du temps proposée par le compositeur à l'imagination inconsciente des auditeurs. Ici, on voit comment un élément sonore ou thématique, répété de manière pratiquement identique en des points opposés du devenir de la forme, prend un sens totalement différent du fait même de sa position dans les successions et les enchaînements. Dans la Cathédrale comme dans la Puerta, tout le début, jusqu'à l'éclatement des sonorités massives de la cathédrale fantôme, reste très sombre: dans ces conditions, la puissance du "choral " central est réellement angoissante, voire menaçante. Or c'est là que, dans la macro-structure, nous voyons le schéma formel amorcé « introduction - allegro " commencer à se dissoudre dans une mouvance dont les contours globaux sont flous, et de plus en plus flous jusqu'à la fin, tout en maintenant des ruptures très tranchées dont l'auditeur ne parvient pas à deviner où elles le conduisent. La forme esquisée, avons-nous dit, s'ouvre et s'estompe : le temps ne mène désormais nulle part, il s'immobilise lentement, tandis que la visions angoissante s'efface et s'éloigne, d'abord dans une sorte d'indifférence ou de neutralité émotionnelle (de 3'08" à 4'02"), puis dans une douceur apaisée et réconfortante (4'02" à la fin). Là est le déni du temps et de la mort, en ce qu'au moment où la forme commence à se désagréger pour l'auditeur, il a le sentiment que le temps s'immobilise (ou s'écoule avec une telle lenteur que cela revient au même), et que la fin du temps de la pièce, la mort de la pièce et ce qu'elle représente, la mort réelle comme horizon existentiel, sont désormais éloignées de lui, dans une sorte de bonheur éternisé. Ainsi, non seulement nous retrouvons, à propos de la Cathédrale, cette dissymétrie formelle qui caractérisait déjà la Puerta, mais nous constatons que le morcellement du temps dans cette dissymétrie fondamentale déséquilibre le climat émotionnel, renvoie le début de la pièce aux 
expériences destructrices inconscientes, puis, en guise de conclusion, provoque leur déni.

2) Mais, nous l'avons également noté, cette signification profonde de toute l'oeuvre de Debussy, qui l'éloigne définitivement de l'ambivalence romantique où le temps intègre la mort comme accomplissement du devenir, s'articule autour des images privilégiées de l'eau. Celles-ci apparaissent nombreuses dans les réponses associées des sujets, alors qu'aucun élément musical analogique ne venait les suggérer.

En réalité, qu'un tel phénomène soit possible - à savoir que la musique de la Cathédrale engloutie suggère immanquablement des images liquides - ne peut se comprendre si on demeure persuadé que la musique « traduit » des impressions visuelles ou sonores, des sentiments ou des émotions. Nous avons insisté jadis (Imberty 1975) sur l'erreur que constitue a priori toute mise en correspondance littérale entre des mots et des rythmes, des accords, des mélodies, des timbres. Significants verbaux et signifiants musicaux ne peuvent être des signifiants équivalents de signifiés identiques. Rappelons que Henri Lefebvre (1966) avait dénoncé fortement cette confusion entre sens et signification, commise encore trop souvent à l'égard d'oeuvres telles que la Cathédrale, dans la mesure où un programme implicite fait illusion. Pour Lefebvre, toute oeuvre musicale a un sens, aucune n'a de signification: l'oeuvre révèle quelque chose qui n'est pas réductible à la seule forme, et qui s'élabore pour l'auditeur lors de l'écoute. Mais c'est cela le sens, qui, lorsqu'il est explicité par des mots, se perd dans les significations verbales, trop précises et trop littérales, et qui le trahissent. Toutes les images particulières et concrètes de l'eau que nous pouvons donc trouver dans les réponses des sujets à propos de la Cathédrale n'ont pas à être prise au pied de la lettre, mais seulement comme indices que quelque sens plus fondamental a été ressenti par les sujets : la Cathédrale, comme la Mer ou Ondine ou Sirènes, ou bien d'autres oeuvres dont le titre n'implique pas forcément l'évocation d'images aquatiques, évoque une eau mythique et fantasmatique, représentation symbolique des fantasmes individuels ou collectifs de la mort et de la peur de la mort.

Tout d'abord, n'oublions pas que l'eau est le thème de toute une époque, celle du symbolisme littéraire dont la vision poétique du monde est comme réfractée à travers les images multiples de l'eau. Le mouvement symboliste, qui cherche à libérer la parole des significations pour retrouver la musique originaire et incantatoire 
des mots, réinvente en fait un des aspects les plus profonds du langage poétique, la " liquidité " de la parole. Cette liquidité est le grand symbole universel de l'harmonie primitive que recherche le poète symboliste dans les sons du langage. Comme l'écrit Bachelard, "l'eau est la maîtresse du langage fluide, du langage sans heurt, du langage continu, continué, du langage qui assouplit le rythme, qui donne une matière uniforme à des rythmes différents " (1948:250). A travers ce nouveau langage, il s'agit de retrouver l'ambiguïté des choses, les impressions fugaces et délicates qu'elles produisent. Dans ce goût pour l'imprécis, le vague et l'impair, c'est-à-dire pour un art ne tranchant jamais à vif dans les significations, mais laissant toujours libre le lecteur de choisir ou de ne pas choisir entre les sensations et les images contradictoires, l'eau apparaît comme le thème privilégié, l'image de toutes les images d'ambiguïté et de double sens. Il est évident que, comme tout l'art de l'époque, la musique de Debussy est imprégnée de cette liquidité, parce que l'imagination du compositeur est elle-même imprégnée des idées des symbolistes. Jarocinski (1966) l'a parfaitement montré.

Il est donc vraisemblable que les auditeurs d'aujourd'hui aient $\mathrm{pu}$ retrouver la structure symbolique inhérente à cette image inconsciente de l'eau, uniquement par la façon dont Debussy dispose les évènements sonores dans le temps musical, organise leurs enchaînements éliptiques, leurs ruptures constantes, leur mobilité fluide, parce que cette image de l'eau a elle-même engendré, dans l'imagination du compositeur, le mouvement sonore qui anime l'écriture. Ce qui ne veut pas dire que la musique de Debussy représente l'eau comme le ferait une marine, mais ce qui veut seulement dire qu'il existe, entre la musique de Debussy et les significations émotionnelles ou mythiques de l'eau des symbolistes, une connivence que l'auditeur peut retrouver à chaque nouvelle audition, sans peut-être pouvoir la nommer, mais qui repose sur le jeu de cette participation, voire de cette confusion, qu'opère l'écoute musicale entre le temps vécu du Moi et le temps musical organisé par le style.

Voilà qui répond aux critiques de Deliège (1984: 32-33) à propos de la méthode des associations verbales pour construire une sémantique musicale. Deliège a raison, tant qu'on s'en tient à ces associations et qu'on n'étudie ni leur structure ni la structure de l'oeuvre musicale. Mais nous avons montré (Imberty 1979 et 1981) que les réseaux associatifs révèlent des structures sousjacentes signifiantes qui se greffent sur les réseaux sonores 
détectés lors de l'audition par le sujet. Dans le langage passe alors l'expérience de ce qui est vécu et reflété dans l'imagination où se scellent les correspondances. Archétypes de l'eau : les archétypes ne sont que des schèmes inconscients, riches de mille potentialités encodables dans les mots, les lignes, les couleurs, les sons. D'ailleurs, puisque Deliège fait référence à la notion bachelardienne de forme concrète, on peut dire que la forme concrète manifeste dans un code les archétypes de l'imagination matérielle.

Or, à propos des Préludes pour le piano, nous avons montré (Imberty 1975, 1976 et 1981) que les connotations de l'eau évoquée par la forme musicale s'organisent autour de trois images archétypiques fondamentales, celles que Bachelard a si bien décrites : l'eau stagnante (ou l'eau morte), l'eau claire, enfin l'eau démontée. L'eau morte et stagnante habite les Brouillards, les Feuilles mortes, plus encore peut-être le désert froid des Pas sur la neige où la glace est l'eau figée. Cette eau "mélancolisante " (Bachelard) est sans doute celle que les sujets perçoivent dans les accords de quinte du début de la Cathédrale, voire dans les sonorités cristallines, mais lointaines et froides qui suivent, sorte d'immobilité inhumaine. L'eau claire, celle des ruisseaux, des sources, de la mer en plein midi habite aussi bien Ondine, Voiles, que Les fées sont d'exquises danseuses ou Bruyères, ou même la piroutte fluide et fantasque de la Danse de Puck. L'eau claire et matinale, c'est aussi certains éclats sonores qui transparaissent dans la grande montée avant l'entrée des accords parfaits parallèles (cette séquence n'est pas de tonalité très sombre, et surtout apparaît très fluctuante du point de vue du climat), c'est surtout l'apaisement central, lorsque la cathédrale s'estompe, et l'apaisement final avec ses sonorités diaphanes. Mais disons tout de suite qu'à la limite, peu importe ce que sont ces sonorités d'ut majeur : nous avons vu comment l'aboutissement de la pièce tout entière est ouverture, esquisse, rêve. Il s'en suit que l'image de l'eau claire correspond profondément à cet aboutissement, que, loin d'être une anecdote dépendante d'un titre ou d'une impression fugitive des auditeurs, elle est représentation temporelle issue de l'évolution de la forme musicale elle-même. L'eau claire est naissance, vie : elle onirise le déni de la mort et l'irréversibilité du temps qui constitue la motivation profonde de l'écriture debussyste. En un sens, presque tous les préludes appellent en leur coda la représentation fantasmatique de l'eau claire ... sauf ceux, peu nombreux, qui, d'un bout à l'autre, témoignent de cette peur panique devant la mort et la destruction universelle. 
Précisément, l'eau démontée, avec ses images de tempêtes, de chaos cosmogoniques, accompagne les pièces les plus violentes et les plus morcelées dans l'écriture du temps : Ce qu'a vu le vent d'ouest, ou plus encore la bourrasque de la partie centrale du Vent dans la plaine témoignent de la prégnance de cette représentation aquatique de la mort. Dans la Cathédrale, ce sont les accords de quinte $f f$, puis la phrase des grands accords qui évoquent ce chaos. Il y a là quelque chose qui peut surprendre : pourtant, les images de la tempête coïncident avec le crescendo qui part de 1'02" (mesure 16) et disparaissent avec la mesure 39, et cette immense progression apparaît comme une menace, laisse percer l'angoisse. En outre, les résonances étendues sur tout le clavier donnent aisément l'impression de diffluence et de profondeur gigantesque qui aident à l'évocation de l'océan. Cependant, là encore, l'important n'est pas que tel ou tel élément musical suggère effectivement par analogie les flots déchaînés : l'eau démontée ne vaut psychiquement que comme image de mort et d'angoisse destructrice, représentation de la difformité, du morcellement de l'univers et du Moi psychique. Or ce n'est pas la première fois que Debussy utilise des accords parallèles pour suggérer la mort : le château " très vieux et très sombre " de Pelléas et Mélisande; les colonnes fantomatiques dans la nuit du temple en ruines (Et la lune descend sur le temple qui fut) ; la douceur mélancolique et étrange qui se dégage des figures funéraires devinées sur la Canope. Dans la Cathédrale engloutie, ce sont ces mêmes accords qui font apparaître la vision inquiétante de la cathédrale morte dans les eaux : le lien entre l'eau démontée et la mort est donc une nouvelle fois très prégnant, si l'on comprend ici que l'eau démontée est d'abord la puissance menaçante de la mort.

3) L'inspiration debussyste est-elle réellement lisible dans l'oeuvre? Les interprétations verbales des auditeurs dépassentelles les clichés culturels ? Bien de nos critiques ont répondu affirmativement à la première question, et nous venons d'essayer de montrer que les interprétations verbales de nos sujets peuvent également aider à retrouver le mouvement de l'imagination créatrice elle-même, à condition de ne pas s'attacher à des littéralités qui ne sont que des façons d'exprimer ce que l'expérience de l'audition a permis de ressentir ou de deviner.

Cependant, comment se fait cette communication du compositeur vers l'auditeur, à travers l'oeuvre? Nous ne pouvons qu'esquisser ici la réponse forcément complexe à cette question (Or la trouvera développée dans Imberty 1981). 
Remarquons que les trois images archétypiques de l'eau, dans la mesure où elles sont sous-tendues par les fantasmes de la mort et du temps, constituent les pôles d'une expérience vitale humaine partagée en partie par toute l'époque symboliste, où l'angoisse métaphysique " rehausse le rang de l'écrivain " (Jarocinski 1966 : 83). La mort et la mer sont partout chez Maeterlinck, comme sont partout chez Baudelaire la mort et le spleen. Le déclin est au goût du jour et les «Français apprennent pour la première fois à prononcer le mot nirvana " (Jankélévitch $1968: 8$ ). Debussy est imprégné de cette atmosphère, lui qui fréquente tous les mardis le salon de Mallarmé. Tout naturellement, il réélabore dans son oeuvre ce qui est à la fois sa propre expérience et celle de ses contemporains. Mais en même temps, il faut reconnaître que l'oeuvre debussyste n'est pas tout à fait à cette humeur élégiaque fin de siècle. Plus exactement, l'eau démontée qui porte la mort violente et destructrice est plus debussyste que ne le sont les délices ouatés du néant. De même, l'eau morte ou stagnante qui porte la mort originelle, le néant absolu d'avant toute vie, a quelque chose de désespéré et glacé qui est plus effrayant que les larmes des séraphins en pleurs de Mallarmé. La Cathédrale engloutie elle-même ne se complaît pas dans l'attirance de l'engloutissement, mais présente une vision plus inquiétante dans sa simplicité et son évidence.

Par conséquent, on peut dire qu'à l'origine de la création artistique, on trouve deux sortes d'archétypes imaginaires : ceux qui proviennent de la culture, ceux qui proviennent des fantasmes inconscients individuels. Les uns et les autres constituent le matériau primitif de l'inconscient et du préconscient de tout individu, à partir duquel s'élaborent les rêves et les oeuvres. Ces archétypes possèdent une généralité et une prégnance psychique exceptionnelles par rapport à toutes les productions oniriques, qui expliquent qu'ils soient reconnus par n'importe quel individu de manière plus ou moins implicite. Ce ne sont pas des images isolées et définitives, mais ce que Guillaumin (1974:225) appelle des "figurations dynamiques ", ou, si l'on veut, des énergies drainant les charges affectives des relations psychiques les plus centrales. À ce titre, les images particulières, visuelles ou sonores, " ne sont que des cas particuliers du symbole, et d'ailleurs elles appellent sans doute en secret tout un ensemble associatif d'images complémentaires préconscientes, prêtes à intervenir dans un ordre plus ou moins rigoureux. De manière générale, ajoute Guillaumin, les recherches contemporaines, notamment dans le domaine du 
langage, ont suffisamment montré que la symbolisation se présentait toujours en réseau, et contextuée, et que chacun de ses termes s'enrichissaient de ses relations avec les autres. "Les processus de symbolisation prennent donc appui sur ces archétypes initiaux (ou symboles archétypiques), mais ceux-ci dépassent la matière visuelle, verbale ou sonore, dans laquelle, à un moment donné, ils s'incarnent, pour prendre leur racine directement dans l'inconscient.

Or les processus psychiques - et surtout les processus inconscients - sont en nombre limité, et se retrouvent toujours les mêmes partout : "représentants-représentations de la pulsion, mécanismes de défense, angoisse, fantasmes " (Anzieu 1970:142). Les symbolisations qu'ils engendrent varient autour de quelques paradigmes représentationnels fondamentaux, directement liés à eux : fantasmes privés lorsqu'ils n'ont aucune forme collective mythique, " mythasmes " lorsqu'au contraire ils sont des condensations de mythes entiers, ou de mythèmes isolés et amalgamés véhiculés par les amplificateurs culturels que sont les langages artistiques, poétiques, musicaux, philosophiques. (La nuit est l'un de ces my thasmes du romantisme, l'eau l'un de ceux du symbolisme littéraire). Toute l'invention créatrice consiste alors à réélaborer ce matériau inconscient ou préconscient commun à toute une culture, universel dans ses éléments les plus archaïques, dans une "gestuelle " stylistique particulière, capable de l'assimiler. Dès lors, le style est inévitablement représentation de ces structures inconscientes, et trouve écho au plus profond de l'inconscient de l'auditeur, du lecteur ou de spectateur. Ou si l'on veut, pour ce qui nous intéresse, dans une production libre de réponses associées, où ce même matériau archétypique est inévitablement utilisé par les sujets, il est normal qu'on retrouve les traces d'un déchiffrement de l'intention stylistique comme représentation du fantasme ou du mythasme sous-jacents. Aux variations personnelles ou situationnelles près, les réponses gravitent autour de ces archétypes représentationnels fondés sur les mécanismes inconscients universels de l'activité représentative symbolique de l'homme.

Ainsi s'éclairerait la relation de sens qui s'établit entre le compositeur, l'oeuvre et l'auditeur : à mi-chemin entre le mythe et le fantasme individuel, lieu privilégié où parfois ils communiquent, l'oeuvre serait pour une part projection du compositeur, pour une part, reflet d'une culture et de ses mythasmes, et, à travers des jeux de miroirs indéfiniment répétés, à travers des 
résonances émotionnelles multipliées, se transmettrait, du compositeur à l'auditeur, le message.

7. Expérience complémentaire : les commentateurs de la Cathédrale ont-ils raison?

1) Le problème.

Si l'on tente de faire le point sur ce qu'on pourrait appeler les épisodes les plus représentatifs ou les plus suggestifs de la Cathédrale engloutie, à travers les descriptions plus ou moins complètes qu'en ont données les critiques musicaux, notamment les descriptions de ceux qui ont cherché à retrouver des épisodes au contenu sémantique précis tout au long de l'oeuvre, en fournissant un lien précis avec la matière sonore, il apparaît que certaines séquences de la pièce sont plus suggestives ou plus immédiatement descriptives - si ce terme a encore un sens après tout ce que nous avons dit - que d'autres. Notamment, le début (mesures 1 à 15), les mesures 28 à 41 (les grands accords parfaits parallèles), et la fin (à partir de la mesure 70) constituent les jalons de l'interprétation sémantique de la Cathédrale par nos auteurs. Cependant, il faut noter que tous ne font pas systématiquement référence à ces trois séquences à la fois, et d'autre part, qu'ils ne leur donnent pas toujours la même signification. Pour certains, le début évoque des eaux transparentes, calmes et statiques ; pour d'autres des eaux glauques et opaques. ${ }^{3}$ Pour leur part, nos sujets ont fort bien perçu le caractère quelque peu ambigu ou trompeur de ces eaux stagnantes, dont le calme apparent masque la mélancolie et la mort (voir le profil, figure 10). Mais les contradictions ou les divergences de signification se retrouvent pour les autres séquences : les grands accords sont un "choral ", un «hymne " pour certains critiques, les piliers de la cathédrale ou l'orgue pour d'autres. De même, la fin est tantôt simple disparition de la vision sous-marine, tantôt l'engloutissement lui-même.

Nous avons donc cherché, dans cette expérience complémentaire, à confronter les descriptions des divers auteurs en question avec les descriptions fournies par des sujets à qui l'on a, au préalable, précisé le titre de l'oeuvre et rappelé le thème de la légende de la ville d'Ys, en soulignant le fait, dans la consigne expérimentale, que la ville, certains jours, transparait au fond des flots, et que Debussy s'est placé de ce point de vue, non du point de vue du récit de l'engloutissement.

La pièce a été au préalable découpée en 10 séquences issues de 
l'expérience précédente et ajustées, pour leur délimitation, sur la partition elle-même. Ces séquences sont les suivantes :

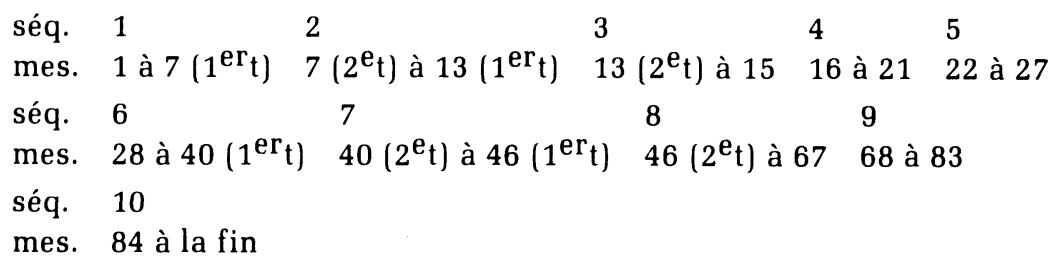

Les sujets (au nombre de trente huit, différents de l'expérience précédente), reçoivent une feuille-réponse divisée en dix parties numérotées correspondant aux séquences ainsi définies. Sur la bande magnétique, un speaker annonce les numéros de séquence en surimpression sur la musique. Les sujets écrivent donc leurs réponses sur leur feuille en suivant l'indication des numéros portés en face de chaque division. La pièce est écoutée une première fois pour simple familiarisation, puis on procède à l'audition expérimentale : celle-ci se déroule sans aucune interruption de bande entre les séquences, et les sujets sont donc invités à donner leurs réponses en suivant le déroulement de la musique.

2) Les résultats

a) Ils font apparaître d'emblée que, malgré la consigne, le titre et les indications de "programme " proposées, huit sujets donnent des réponses totalement indépendantes du thème légendaire. Leurs réponses, toutes sémantiques (donc pas de réponses formelles) n'apportent aucun élément nouveau par rapport à l'expérience précédente. Par conséquent, on peut dire que le titre et la connaissance de la légende ne constituent pas des éléments nécessaires pour l'interprétation de la Cathédrale, ou, plus exactement, qu'ils n'apparaissent pas comme indispensables et pertinents pour un nombre non négligeable de sujets. Ces mêmes sujets donnent par ailleurs des réponses qui entrent parfaitement dans le cadre plus général défini par les associations spontanées libres.

b) Treize sujets donnent des réponses qui ne concernent que partiellement la Cathédrale et la légende de la ville d'Ys. Les aspects retenus sont d'abord ceux qui concernent la mer et, d'une façon générale les images de l'eau. Mais on remarque que, pour le même nombre de sujets, les réponses aquatiques sont en nombre équivalent dans cette expérience et la précédente, où ni le titre ni la légende ne pouvaient influencer les sujets. On retrouve donc l'importance de l'eau dans l'imaginaire de Debussy. Les autres 
aspects de la légende retenus sont les cloches, reconnues mais de manière non localisée, et l'engloutissement de la cathédrale ou sa disparition à la fin, malgré la consigne qui précisait bien que la pièce ne racontait pas la fin de la ville d'Ys, mais en évoquait la vision à travers les flots marins : cet engloutissement s'impose dès la disparition des grands accords, au moment où les connotations de tristesse et de mort font leur apparition (séquences 7, 8 et 9). La vision de la ville est ce qui est le moins suggéré par ceux qui n'ont tenu compte que d'une partie de la consigne : sans doute, et contrairement à ce que ceux qui connaissent trop l'oeuvre pensent, les grands accords n'ont pas immédiatement cette massivité de la pierre et des ruines. Par contre, une nouvelle fois, comme nous l'avons montré dans l'expérience précédente, les images de l'eau démontée s'imposent plus facilement.

c) Dix sept sujets cherchent à greffer un récit ou le déroulement d'une vision à travers les dix séquences qui leur sont proposées. Par rapport aux sujets précédents, les aspects plus spécifiques de la légende apparaissent mieux : les cloches sont mieux localisées (séquences 3,4 et 7), l'apparition de la cathédrale ou de la ville engloutie (séquences 5, 6 et 9); un chant ou un orgue ou un office religieux sont perçus à la séquence 6 (les grands accords !) ; enfin l'engloutissement ou la disparition, l'anéantissement de la vision ou de la ville elle-même dominent aux séquences $6,7,8$ et 9 , avec des connotations de mort et d'angoisse plus marquées que chez les sujets précédents. L'ensemble de ces constatations est résumé dans le tableau IV.

d) Tous les autres éléments qui apparaissent comme des compléments précisant l'at mosphère lumineuse, ou l'heure du jour, demeurent des réponses très individuelles : ceux des critiques qui ont éprouvé le besoin de décrire des jeux de lumière sur l'eau ont donc largement dépassé ce que suggère la musique elle-même. Il est également curieux de constater que la brume est assez peu évoquée, soit qu'elle se lève (comme l'indique Debussy, au début de la séquence 4), soit au contraire qu'elle tombe (à la fin en particulier). Poétiquement, cette indication de Debussy sur la partition serait surtout une aide à l'interprétation, invitant le pianiste à jouer sur autre chose que le tempo (timbre du piano, jeux de la pédale, etc... ). 


\begin{tabular}{|c|c|c|c|c|c|c|c|c|c|c|}
\hline Séquences & 1 & 2 & 3 & 4 & 5 & 6 & 7 & 8 & 9 & 10 \\
\hline \multirow[t]{2}{*}{ mer, eau, pluie } & 11 & 8 & 4 & 6 & 5 & 4 & 7 & 6 & 9 & 4 \\
\hline & 7 & 5 & 6 & 10 & 6 & 6 & 2 & 2 & 6 & 0 \\
\hline \multirow[t]{2}{*}{ cloches } & 0 & 2 & 3 & 6 & 3 & 2 & 3 & 2 & 1 & 2 \\
\hline & 2 & 2 & 1 & 1 & 1 & 1 & 2 & 1 & 1 & 0 \\
\hline \multirow{2}{*}{$\left.\begin{array}{l}\text { brume (apparition } \\
\text { ou disparition) }\end{array}\right\}$} & 1 & 1 & 1 & 2 & 3 & 3 & 1 & 1 & 1 & 0 \\
\hline & 2 & 0 & 0 & 1 & 1 & 0 & 2 & 2 & 0 & 0 \\
\hline \multirow[t]{2}{*}{ lumière, aube, jour } & 1 & 1 & 1 & 0 & 0 & 1 & 0 & 0 & 0 & 0 \\
\hline & 1 & 3 & 0 & 1 & 2 & 2 & 0 & 1 & 1 & 0 \\
\hline \multirow[t]{2}{*}{ nuit } & 1 & 0 & 0 & 0 & 0 & 0 & $\mathbf{0}$ & 0 & 3 & 1 \\
\hline & 2 & 0 & 0 & 0 & 0 & 0 & 2 & 3 & 1 & 0 \\
\hline \multirow[t]{2}{*}{ église, ville, château } & 2 & 1 & 0 & 3 & 8 & 7 & 5 & 3 & 4 & 1 \\
\hline & 0 & 0 & 0 & 0 & 1 & 0 & 0 & 2 & 1 & 1 \\
\hline \multirow{2}{*}{$\left.\begin{array}{l}\text { orgue, chant, procession, } \\
\text { office religieux }\end{array}\right\}$} & 1 & 0 & 0 & 0 & 3 & 4 & 0 & 0 & 1 & 0 \\
\hline & 0 & 0 & 0 & 0 & 0 & 3 & 0 & 1 & 1 & 0 \\
\hline \multirow{2}{*}{$\left.\begin{array}{l}\text { tristesse, mort, } \\
\text { engloutissement, angoisse }\end{array}\right\}$} & 2 & 1 & 1 & 1 & 3 & 2 & 8 & 5 & 8 & 3 \\
\hline & 0 & 0 & 0 & 2 & 1 & 0 & 3 & 6 & 6 & 1 \\
\hline \multirow[t]{2}{*}{ calme, douceur, sérénité } & 7 & 1 & 0 & 0 & 0 & 2 & 1 & 4 & 4 & 4 \\
\hline & 6 & 1 & 1 & 0 & 3 & 2 & 10 & 3 & 0 & 6 \\
\hline
\end{tabular}

\section{Tableau IV}

Principaux sémantèmes associés à la Cathédrale engloutie, par des sujets connaissant le titre et la légende de la ville d'Ys.

$$
\text { Valeur critique }=5(P=.02)
$$

Pour chaque sémantème : la première ligne concerne les réponses fournies par les sujets ayant tenu complètement compte de la consigne ; la seconde ligne concerne les résultats de ceux qui n'ont retenu que certains aspects de la consigne. 
e) Deux conclusions s'imposent à travers ces faits. La première est que les éléments suggestifs de la pièce, ceux qui "imiteraient " les cloches, l'orgue ou la massivité de la cathédrale, l'engloutissement dans les flots, ne sont seulement lisibles que si le titre vient induire les associations sémantiques, mais encore que si l'auditeur lui-même est prêt à jouer le jeu. À cet égard, ceux des sujets qui n'ont pas tenu compte des indications qui leur étaient fournies, ont opéré un choix qui les empêchait de décrypter ces imitations soit-disant évidentes et naturelles. On retrouve là, en ce qui concerne les procédés imitatifs ou descriptifs dont dispose la musique, cette dimension conventionnelle dont l'origine est souvent historique ou culturelle, transmise par l'éducation ou les idées à la mode, et sans lesquelles ces procédés eux-mêmes n'auraient plus aucun pouvoir. Ce qui ne veut pas dire qu'en l'occurrence, le titre de Debussy soit arbitraire : toute convention peut être motivation à partir du moment où l'auditeur en connaît l'origine. Le figuralisme musical ne date pas d'hier, et la plupart de ses procédés sont pourtant largement conventionnels.

On aboutirait en définitive ici aux correspondances suivantes :

- le calme, l'immensité marine, l'horizon vide et la profondeur $=$ les accords de quinte ;

- les cloches = le motif tritonique si bémol, do, fa - si bémol, do sol, et ses variantes depuis la mesure 16, qui évoquent traditionnellement les carillons depuis le $\mathrm{XIX}^{\mathrm{e}}$ siècle ;

- la massivité de la cathédrale, les ruines de la ville = les accords parfaits parallèles, invention debussyste, comme on l'a vu plus haut, mais qui ne serait pas sans évoquer les accords parfaits de la grande porte de Kiev des Tableaux d'une exposition de Moussorgsky, dont Debussy se serait souvenu (?) ;

- les effets de pédale sur les ostinato graves = l'engloutissement.

Mais la seconde conclusion est que l'attitude qu'impliquait chez nos sujets la présente expérience, est elle-même différente de celle exigée lors de l'expérience précédente. Il s'agissait ici de trouver des correspondances entre une oeuvre musicale déroulée dans sa durée et un récit qu'ils avaient à reconstruire à partir du titre et de la consigne expérimentale. Or la conduite du récit est elle-même temporelle, et suppose des cadres a priori de mise en ordre des évènements contenus dans ce récit, cadres qui constituent justement une macro-structure. Celle-ci, nous l'avons vu, se présente comme un ensemble d'entrées vides, où viennent se 
loger les segments du récit. Ceci pourrait expliquer une contradiction entre l'analyse sémantique du début de la Cathédrale, tel qu'il apparaissait précédemment (eau stagnante, eau mélancolisante), et l'impression initiale de paix et de sérénité qu'inspire ici ce même début, à travers les images du grand large.

En effet, on pourrait suggérer que la demande de récit légendaire a situé d'emblée les sujets dans un cadre temporel conventionnel défini, celui du "il était une fois ... » ou du " par un beau matin de printemps breton...». Bref, les sujets sont entrés dans ce cadre du récit qui implique quelque chose comme une mise en exposition, un développement avec une crise, un dénouement. Du coup, la verbalisation demandée a pu influer sur la perception de la forme musicale elle-même, en donnant au début de la pièce ce rôle de simple exposition, ce qui, du même coup, lui a fait perdre une partie de sa charge affective immédiate. L'hypothèse est d'autant plus vraisemblable qu'à propos de la Puerta del Vino, nous avions noté (Imberty 1981) que certains sujets, lorsqu'on leur demandait, après audition, comment ils décriraient (verbalement) la pièce qu'ils venaient d'entendre, proposaient une description en trois parties ( $\mathrm{ABA}$ ), en oubliant carrément les quatre premières mesures, puisqu'ils faisaient commencer la pièce de manière " douce, sombre et mélodieuse ". Les structures générales par lesquelles nous racontons des histoires sont des structures de mise en ordre du temps, selon certains schémas culturels stéréotypés, et on peut donc penser qu'ici, la consigne a induit sur la musique certains aspects de ces structures générales.

De ce fait, les critiques ont souvent mentionné le caractère limpide et heureux du début de la Cathédrale engloutie. Peut-être, est-ce surtout imputable à l'at titude de récit qu'ils ont adoptée, car, nous l'avons vu, le début de cette célèbre pièce apparaît relativement plus sombre, troublant, tout au moins ambigu (figure 10) en audition libre.

Méfions-nous donc des commentaires trop littéraires qui masquent toutes les richesses troublantes et indéfiniment interprétables de la partition! 


\section{NOTES}

1. Les nombres d'unités de la base des arbres hiérarchiques ne doivent pas être confondus avec les nombres de changements perçus pour chaque pièce : en effet les figures 1 et 2 sont construites seulement à partir des changements perçus de manière relativement stable et non à partir de tous les changements indiqués par les sujets. Il n'en reste pas moins que même à ce niveau, la « densité " de la pièce de Debussy est plus grande que celle de Brahms ( 28 u./ $175 \mathrm{sec}$. , et $327 . / 271 \mathrm{sec}$.).

2. Nous voulons dire par là : non repérable directement au niveau neutre dans des unités à même le texte écrit.

3. Nous nous appuyons sur un relevé inédit, amicalement communiqué par J.-J. Nattiez.

\section{RÉFÉRENCES}

ANZIEU, D.

1970 : «Freud et la mythologie », Nouvelle revue de psychanalyse, I, 114-45.

BACHELARD, G.

1942 : L'eau et les rêves. Paris : Corti.

DELIĖGE, C.

1984 : Les fondements de la musique tonale. Paris : Lattès.

FRAISSE, $P$.

1957 : Psychologie du temps. Paris : Presses Universitaires de France.

FRANCÈS, R.

1958 : La perception de la musique. Paris : Vrin. GUILLAUMIN, J.

1974 : "La création artistique et l'élaboration consciente de l'inconscient, avec des considérations particulières sur la création poétique ", in Anzieu, D., éd., Psychanalyse du génie créateur. Paris : Dunot, 209-37.

IMBERTY, M.

1974 : "Intégration formelle et pouvoir impressif de l'oeuvre musicale », Sciences de l'Art - Scientific Aesthetics, IX/1-2, 15-32.

1975 : "Perspectives nouvelles de la sémantique musicale expérimentale », Musique en jeu, $\mathrm{N}^{0} 17,87-109$. 
1976 : «Polysémie et cohérence du langage musical. - III. Représentation sémantique du style ", Sciences de l'Art - Scientific Aesthetics, /2, 139-59.

1976 : Signification and Meaning in Music. Montréal : Université de Montréal, Monographies de sémiologie et d'analyses musicales.

1977 : "Structure perceptive du style musical ", Bulletin de psychologie, 329, 30, (14-16), 781-9 (= Sixième colloque international d'esthétique expérimentale, Paris, 1976).

1979 : Entendre la musique. Paris : Dunot.

1981 : Les écritures du temps. Paris : Dunod.

JANKÉLÉVITCH, V.

1968 : La vie et la mort dans la musique de Debussy. Neuchâtel : La Baconnière.

\section{JAROCINSKY, J.}

1970 : Debussy, impressionnisme et symbolisme. Paris : Seuil.

KINTSCH, W, et VAN DIJK, A.

1975 : "Comment on se rappelle et on résume des histoires", Langages, $\mathrm{N}^{0} 40,98-116$.

\section{LEFEBVRE, $\mathrm{H}$.}

1966 : Le langage et la société. Paris : Gallimard.

LÉVI-STRAUSS, C.

1972 : L'homme nu. Paris : Plon.

NATTIEZ, J.-J.

1975 : Fondements d'une sémiologie de la musique. Paris : Union Générale d'Éditions. Collection 10-18.

PIAGET, J.

1962 : Les mécanismes perceptifs. Paris : Presses Universitaires de France.

RUWET, N.

1972 : Langage, musique, poésie. Paris : Seuil.

1975 : «Théories et méthodes dans les études musicales : quelques remarques rétrospectives et préliminaires ", Musique en jeu, $\mathrm{N}^{0} 17,11-36$. 